\title{
Lévy noise-induced self-induced stochastic resonance in a memristive neuron
}

\author{
Marius E. Yamakou(i) • Tat Dat Tran $(\mathbb{D}$
}

Received: 20 August 2021 / Accepted: 20 November 2021 / Published online: 6 December 2021

(C) The Author(s) 2021

\begin{abstract}
All previous studies on self-induced stochastic resonance (SISR) in neural systems have only considered the idealized Gaussian white noise. Moreover, these studies have ignored one electrophysiological aspect of the nerve cell: its memristive properties. In this paper, first, we show that in the excitable regime, the asymptotic matching of the deterministic timescale and mean escape timescale of an $\alpha$-stable Lévy process (with value increasing as a power $\sigma^{-\alpha}$ of the noise amplitude $\sigma$, unlike the mean escape timescale of a Gaussian process which increases as in Kramers' law) can also induce a strong SISR. In addition, it is shown that the degree of SISR induced by Lévy noise is not always higher than that of Gaussian noise. Second, we show that, for both types of noises, the two memristive properties of the neuron have opposite effects on the degree of SISR: the stronger the feedback gain parameter that controls the modulation of the membrane potential with the magnetic flux and the weaker the feedback gain parameter that controls the saturation of the mag-
\end{abstract}

M. E. Yamakou $(\varangle)$

Department of Data Science,

Friedrich-Alexander-Universität Erlangen-Nürnberg,

Cauerstr. 11, 91058 Erlangen, Germany

e-mail: marius.yamakou@fau.de

T. D. Tran $(\varangle)$

Fakultät für Mathematik und Informatik, Universität Leipzig, Augustusplatz 10, 04109 Leipzig, Germany

e-mail: tran@math.uni-leipzig.de

M. E. Yamakou · T. D. Tran

Max-Planck-Institut für Mathematik in den Naturwissenschaften, Inselstr. 22, 04103 Leipzig, Germany netic flux, the higher the degree of SISR. Finally, we show that, for both types of noises, the degree of SISR in the memristive neuron is always higher than in the non-memristive neuron. Our results could guide hardware implementations of neuromorphic silicon circuits operating in noisy regimes.

Keywords Slow-fast dynamical systems - Lévy noise $\cdot$ Self-induced stochastic resonance $\cdot$ Memristive neuron

\section{Introduction}

Noise is ubiquitous in neural systems and several studies have shown that it can play a constructive role in information processing [1-10]. Noise-induced resonance mechanisms are a category of phenomena showing this constructive counter-intuitive role of noise. Several types of noise-induced resonance mechanisms have been identified and extensively studied, particularly in neural systems. These include stochastic resonance (SR) [1,4,11-14], coherence resonance (CR) [5,15-18], spatial CR $[19,20]$, inverse stochastic resonance [21-25], recurrence resonance [26], and selfinduced stochastic resonance (SISR) [25,27-35].

All previous investigations on SISR have treated the input noise process as solely Gaussian [25,27-35]. But stochastic processes with a Lévy distribution are wellknown to more accurately model the dynamics of real biological neurons [36,37]. In general, dynamical sys- 
tems composed of a large number of nonlinearly coupled subsystems often obey the Lévy distribution [3840]. Thus, in neural systems, the Lévy distribution on the network level reflects the emergent properties of the network in which the neurons are the subsystems. And at the level of the individual neuron, this implies that it is also composed of nonlinearly coupled subsystems - the ionic channels. In [41], a plot of the interspike intervals and the interevent intervals distributions indicates that neurons and neural network activities are characterized by a non-Gaussian heavy-tail interval distribution, thereby providing a solid reason as to why it makes sense to consider Lévy noise in the study of neural systems. Lévy noise has also been extensively used to model many other complex systems, including lasers [42], quantum dots [43], cardiac dynamics [38], molecular motor [44], economics [45,46], and social systems [47], where changes are often abrupt [48,49]. Furthermore, several studies on stochastic systems have departed from Gaussian to Lévy processes and compared their effects. For example, in [47], the study of the stochastic payoff variations in the spatial prisoner's dilemma game is presented; in [50], the neuron competition models; and in [51], the statistical complexity and normalized Shannon entropy of the FitzHugh-Nagumo neuron model. Motivated by the relevance of Lévy processes in neural systems explained above, this paper focuses on SISR in a memristive neuron perturbed by a Lévy noise-a setting that has not been considered before. We obtained the analytical conditions required for the occurrence of SISR and the parameters combination of the Lévy noise that maximize the degree of SISR. We then compare these analytical conditions and the degree of SISR to those of Gaussian noise.

Generically, SISR occurs when a multiple-timescale excitable dynamical system is driven by a weak noise amplitude. During SISR, the escape timescale of trajectories from one attracting region in phase space to another is distributed exponentially, and the associated transition rate is governed by an activation energy. Suppose the excitable system (e.g., a neuron) is placed out-of-equilibrium, and its activation energy decreases monotonically as the neuron relaxes slowly to a stable quiescent state (stable fixed point); then, at a specific instant during the relaxation, the timescale of escape due to noise and the timescale of relaxation match, and the neuron fires at this point almost surely. If this activation brings the neuron back out-of-equilibrium, the relaxation stage can start over again, and the scenario repeats itself indefinitely, leading to a coherent spiking activity which cannot occur without noise. SISR essentially depends on the interplay of three different timescales: the slow and fast timescales in the deterministic equation of the system, plus a third timescale characteristic to the noise.

It is important to note that the mechanism of SISR is very different from those of SR and CR. In fact, it has been shown in [28] that CR and SISR are actually two distinct mechanisms even though both lead to the emergence of weak noise-induced coherent oscillations. Moreover, in our previous work [34] (see also [52]), it has been shown that the way SISR in the first layer of a duplex neural network controls CR in the second layer is different from the control of CR when we have CR in the first layer. This difference in the controllability of CR by SISR and CR in multiplex networks further confirms the fact that CR and SISR are actually different mechanisms. Compared to CR and SR, the conditions to be met for the mechanism of SISR are more subtle: Like CR, SISR does not require an external periodic signal as in SR. Remarkably, unlike CR, SISR does not require the system's parameters to be in the vicinity of bifurcation thresholds, making it more robust to parametric perturbations than CR. Moreover, unlike both SR and CR, SISR requires a strong timescale separation between the variables of the excitable system.

The exchange of charged ions across the membrane of the nerve cell can induce complex electromagnetic field inside and outside this membrane, and the membrane potential of neuron gets modulated by the induced electromagnetic field. Thus, by Faraday's law of electromagnetic induction, the effect of electromagnetic induction on the cell must be considered. Recently, Lv et al. [53] proposed a modified neural model that takes into account the effect of the magnetic field generated by the internal bioelectricity of the nerve cell (i.e., the movement of charged ions across the membrane on the spiking activity of the cell). In the modified (improved) neuron models, the effects of electromagnetic induction are described by using the magnetic flux. And the modulation of the membrane potential by the magnetic flux is realized by using a memristor coupling, hence the term memristive neurons [54]. The modification of the original neural models such that they take into account these electromagnetic effects, consisted of adding a variable for the magnetic flux into the original equations. 
Several studies have shown that memristive neurons can generate a rich variety of modes in electric activities by not only varying the external input current, but also by varying the magnetic flux parameters-those that control the memristive properties of the neuron [55-60]. It has been shown that the magnetic flux coupling between neurons [57] and other nonlinear circuits $[61,62]$ can induce perfect phase synchronization of chaotic time series of oscillations. The effect of the memristive properties of neurons on the stability of their synchronization has also been demonstrated [63]. In [64], it is shown that appropriate intensity of electromagnetic radiation could be effective to realize intermittent synchronization, while stronger intensity of electromagnetic radiation can induce disorder of coupled neurons and network. It was also demonstrated that the electromagnetic induction strength combined with an asymmetric electrical coupling and external stimulus induce the co-existence of bifurcations and multiple (chaotic and periodic) firing patterns in coupled neural oscillators [65]. Furthermore, PSpice-based simulations have also confirm that the electrical activities and synchronization between coupled neurons can be modulated by the electromagnetic flux $[66,67]$.

It has also been shown that the magnetic field coupling can contribute to the signal exchange between neurons by triggering superposition of electric field when synapse coupling is not available [58]. Here, the contribution of the field coupling from each neuron is described by introducing appropriate weight dependent on the distance between two neurons. It was found that the degree of synchronization is dependent on the intensity and weight of the field coupling and that the pattern selection of the network connected with gap junction can be modulated by this field coupling.

The memristive properties have also been shown to play a significant role in the dynamics of other types of biological tissues. For example, it has been shown that target wave propagation can be blocked to stand in a local area of the cardiac tissue and the excitability of this tissue can be suppressed to approach quiescent but homogeneous state when an electromagnetic flux (generated by the motion of ions across the membrane of the cardiac cell) is imposed on the cardiac tissue [59]. Moreover, it has been shown that a spiral wave can be triggered and developed by setting specific initial conditions in the cardiac tissue under the effects of magnetic flux, i.e., the tissue still support the survival of standing spiral waves under specific values of the magnetic flux parameters [60].

In [68], the electromagnetic induction due to the memristive properties of the neurons has been shown to play important roles in the regulation of sleep wake cycle, where the time of wake up is delayed and fall asleep is advanced when the electromagnetic induction and its noise are considered. Furthermore, the effects of electromagnetic noise on the regulation have been shown to inhibit the neuronal discharge activities and change the time of wake up and fall asleep of the neurons.

It is now well-accepted that the effects of the magnetic flux across the membrane of the cell should be considered when investigating the emergence of electrical activities and wave propagation in the nerve and cardiac cells $[53,59]$. However, all previous studies on SISR in neural systems have been done only with nonmemristive models perturbed by Gaussian noise. Thus, the effect of the memristive properties of a neuron on Lévy and Gaussian noise-induced SISR are still elusive. In this paper, we bridge this gap by applying nonlinear dynamics methods and numerical simulations to address the following questions: (i) Can Lévy noise (with polynomial intrinsic timescale) also induce SISR? (ii) Which noise induces the highest degree of SISR, Lévy or Gaussian noise? (iii) How do the memristive properties of the neuron affect the degree of SISR induced by these two types of noises?

The rest of the paper is organized as follows: In Sect. 2, we describe the mathematical equation modelling a memristive neuron driven by Lévy noise and we also determine the excitable parameter space of model in terms of the memristive parameters. Section 3 is devoted to the theoretical analysis of the mechanism of SISR. In Sect. 4, we present and discuss the numerical results. And in Sect. 5, we have summary and conclusions.

\section{Mathematical model and excitability}

\subsection{Model description}

We consider a memristive FitzHugh-Nagumo (FHN) neuron model of type-II excitability [53,69], driven by an $\alpha$-stable Lévy process, and described by the following stochastic differential equations 


$$
\left\{\begin{array}{l}
d v_{\tau}=\varepsilon^{-1} f_{1}\left(v_{\tau}, w_{\tau}, \phi_{\tau}\right) d \tau+\frac{1}{\sqrt[\alpha]{\varepsilon}} d L^{\alpha, \beta}(\tau ; \sigma, \mu), \\
d w_{\tau}=f_{2}\left(v_{\tau}, w_{\tau}, \phi_{\tau}\right) d \tau \\
d \phi_{\tau}=f_{3}\left(v_{\tau}, w_{\tau}, \phi_{\tau}\right) d \tau
\end{array}\right.
$$

with the deterministic velocity vector field given by

$$
\left\{\begin{array}{l}
f_{1}(v, w, \phi)=v-\frac{v^{3}}{3}-w-k_{1} \rho(\phi) v \\
f_{2}(v, w, \phi)=v+d-c w \\
f_{3}(v, w, \phi)=v-k_{2} \phi
\end{array}\right.
$$

where $(v, w, \phi) \in \mathbb{R}^{3}$ represent the action potential variable $v$, the recovery current (or sodium gating) variable $w$ that restores the resting state of the neuron, and the third variable $\phi$ is the magnetic flux across membrane which can generate additive current.

The parameter $0<\varepsilon:=\tau / t \ll 1$ is the timescale separation ratio (also called singular parameter) between the slow timescale $\tau$ and the fast timescale $t$. It accounts for the slow kinetics of the sodium channel in the nerve cell and controls the main morphology of the action potential generated [70]. It is worth noting that $\varepsilon$ is a very small and positive parameter $(0<\varepsilon \ll 1)$, and from Geometric Singular Perturbation Theory (GSPT) for slow-fast dynamical systems in the standard form [71], this means that the $v$-variable is fast and the $w$ - and $\phi$-variables are slow. Moreover, from GSPT, the relation $\varepsilon:=\tau / t$ can be used (i.e., $d \tau:=\varepsilon d t$ ) to transform Eq. (1) from the slow timescale $\tau$ to the fast timescale $t$, given by Eq. (14). We further note that Eqs. (1 and 14) are equivalent except that their orbits evolve on different timescales. The constant parameter $d$ is such that $d \in(0,1)$, and $c>0$ is a codimension-one Hopf bifurcation parameter.

The term $\rho(\phi)$ in Eq. (2) is the memory conductance of a magnetic flux-controlled memristor and it is used to describe the coupling between magnetic flux $\phi$ and membrane potential $v$ of the neuron [72-74]. The memory conductance of a memristor is often described by

$\rho(\phi)=a+3 b \phi^{2}$,

where $a$ and $b$ are constant parameters. In this paper, we fix $a=0.1$ and $b=0.02$, to stay consistent with other works [75]. The magnetic feedback gain parameters $k_{1}$ and $k_{2}$ describe the interaction between the magnetic flux and membrane potential. More precisely, $k_{1}$ bridges the coupling and modulation on the membrane potential $v$ from magnetic flux $\phi$, and $k_{2}$ describes the degree of polarization and magnetization by adjusting the saturation of magnetic flux [76]. The term $k_{1} \rho(\phi) v$ in Eq. (2), therefore, describes the modulation on the membrane potential of the neuron, and it depends on the variation in the magnetic flux. Combining Faraday's law of electromagnetic induction and the basic properties of a memristor, the term $k_{1} \rho(\phi) v$ is regarded as additive induction current on the membrane potential. The dependence of electric charge $q$ on the magnetic flux $\phi$ is defined as [77]

$\rho(\phi)=\frac{d q(\phi)}{d \phi}=a+3 b \phi^{2}$.

Moreover, because the current $i$ is defined as the time derivative of charge $q$, the physical significance for the term $\rho(\phi) v$ could be described as

$i=\frac{d q(\phi)}{d t}=\frac{d q(\phi)}{d \phi} \frac{d \phi}{d t}=\rho(\phi) V=k_{1} \rho(\phi) v$,

where $V$ denotes an induced electromotive force with a feedback gain parameter $k_{1}$. The potassium and sodium ionic currents contribute to the magnetic flux across the membrane and also to the membrane potential. This introduces a negative feedback term $-k_{2} \phi$ in the third equation of Eq. (2).

$L^{\alpha, \beta}(\tau ; \sigma, \mu)$ is an independent $\alpha$-stable Lévy motion. The Lévy motion, as an appropriate model for non-Gaussian processes with jumps [78,79], has properties of stationary and independent increments. Throughout this paper, we adhere to one of the possible parametrizations of $\alpha$-stable distributions [80,80-82] which allows to write down the characteristic function of an appropriate probability distribution

$\phi(x)=\int_{-\infty}^{\infty} e^{-i x \zeta} L^{\alpha, \beta}(\zeta ; \sigma, \mu) d \zeta$,

in the form of

$\phi(x)=\exp \left[i \mu x-\sigma^{\alpha}|x|^{\alpha}\left(1-i \beta \operatorname{sgn}(x) \tan \frac{\pi \alpha}{2}\right)\right]$,

if $\alpha \in(0,1) \bigcup(1,2]$, or

$\phi(x)=\exp \left[i \mu x-\sigma|x|\left(1+i \beta \frac{2}{\pi} \operatorname{sgn}(x) \ln |x|\right)\right]$,

if $\alpha=1$. Here, $\alpha$ stands for the stability index and lies in the interval $\alpha \in(0,2]$. It describes an asymptotic power law of the $\zeta$-distribution, $L^{\alpha, \beta}(\zeta ; \sigma, \mu) \sim$ $|\zeta|^{-(\alpha+1)}$, and controls the impulsiveness (i.e., the jump frequency and size) of the process. The parameter $\beta \in[-1,1]$ determines the skewness (asymmetry) 

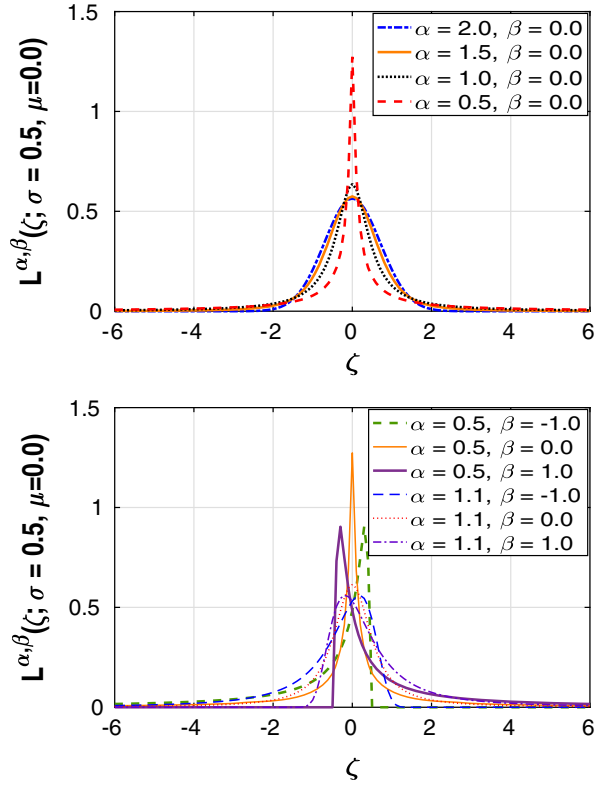

Fig. 1 Probability density functions for Lévy distribution of $L^{\alpha, \beta}(\zeta ; \sigma=0.5, \mu=0.0)$ with different values of the stability index and skewness parameters

of the distribution. $\sigma \in(0, \infty)$ is the scale parameter. $\mu \in \mathbb{R}$ is the location parameter. Closed, analytical forms of the stable Lévy probability densities are known in some cases. For example, $L^{2,0}(\cdot ; \sigma, \mu)$ is the well-known Gaussian distribution; $L^{1,0}(\cdot ; \sigma, \mu)$ yields the Cauchy distribution; $L^{\frac{1}{2}}, 1(\cdot ; \sigma, \mu)$ yields the LévySmirnoff $(\zeta>\mu)$ distribution; and other forms can be found in $[83,84]$.

Figure 1 shows the probability density functions of the Lévy distribution $L^{\alpha, \beta}(\zeta ; \sigma, \mu)$ with some values of the stability index and skewness parameters. Throughout this paper, we fix the location parameter at $\mu=0.0$ and use the notations $L^{\alpha, \beta}(\zeta), L(\zeta)$, and $L_{\zeta}$, interchangeably.

\subsection{The excitable regime of the model}

In this subsection, we determine the excitable regime of the memristive FHN neuron model in terms of the Hopf bifurcation $c$ and memristive parameters $k_{1}$ and $k_{2}$. The deterministic memristive FHN neuron (i.e., Eq. 1 without the noise term) with a unique and stable fixed point cannot maintain a self-sustained spiking activity. One says in this case that the neuron is in the excitable regime [85], in contrast to the oscillatory regime, where the neuron continuously spikes due to the occurrence of a bifurcation onto a limit cycle. In the excitable regime, choosing an initial condition in the basin of attraction of this unique and stable fixed point will result in at most one large non-monotonic excursion into the phase space after which the trajectory returns to this fixed point and stays there until the initial conditions are changed again.

The deterministic predisposition required for SISR is an excitable regime, so that during SISR, the selfsustained and coherent spiking produced by the neuron is due solely to the presence of noise and not because of the occurrence of bifurcations onto a limit cycle. This is one of the crucial differences between SISR and $\mathrm{CR}$ - the predisposition required for the latter mechanism is the close proximity of parameters to the bifurcation threshold, so that weak noise amplitudes can easily drive the system to this bifurcation threshold without, stochastically, overwhelming the dynamics $[15,17,28]$.

It is worth noting that even though nonlinear systems such as the one considered in this work can admit multistability [23], hidden firing patterns [65,86], or Hopf bifurcation, we do not consider any of these regimes because we are interested in the phenomenon of SISR that requires an excitable regime and via which the system exhibits coherent spiking induced solely by noise. For the sake of brevity and conciseness, in this work we only discuss the stability (in terms of the Hopfbifurcation parameter $c$ and the memristive parameters $k_{1}$ and $k_{2}$ ) of the unique fixed point which sets the model into an excitable regime required for SISR. We refer the reader to $[23,87-90]$ if there is an interest in a full bifurcation analysis of the FHN neuron model.

At the fixed points $\left(v_{e}, w_{e}, \phi_{e}\right) \in F i x$ (the set of rest states of the neuron), the variables $v(\tau), w(\tau)$, and $\phi(\tau)$ reach a stationary state, while the set of fixed points defined by the intersection of the nullclines as

$$
\text { Fix }:=\left\{(v, w, \phi) \in \mathbb{R}^{3}: f_{1}=f_{2}=f_{3}=0\right\},
$$

depends on the parameters $c, d, k_{1}$, and $k_{2}$. The sign of

$\Delta=\frac{g^{2}}{4}+\frac{p^{3}}{27}$

determines the number of fixed points. In this paper, we consider the case where we have only one stable fixed point. If $\Delta>0$, we have a unique fixed point given by 
$\left\{\begin{array}{l}v_{e}=\sqrt[3]{-\frac{g}{2}-\sqrt{\Delta}}+\sqrt[3]{-\frac{g}{2}+\sqrt{\Delta}} \\ w_{e}=\frac{1}{c}\left(v_{e}+d\right), \\ \phi_{e}=\frac{v_{e}}{k_{2}}\end{array}\right.$

where

$$
\left\{\begin{array}{l}
p=\frac{\frac{1}{c}+k_{1} a-1}{\frac{1}{3}+\frac{3 k_{1} b}{k_{2}^{2}}}, \\
g=\frac{\frac{d}{c}}{\frac{1}{3}+\frac{3 k_{1} b}{k_{2}^{2}}} .
\end{array}\right.
$$

Moreover, in the model we arbitrarily fix $d=0.5$ once and for all, and we determine the excitable regime of the model in terms of the parameter $c$ and the two new parameters $k_{1}$ and $k_{2}$-also known as the magnetic gain parameters. With the fixed values of the parameters $a=0.1, b=0.02$, and $d=0.5, p$ and $g$ in Eq. (12) now depend only on $c, k_{1}$, and $k_{2}$. We have:

$$
\left\{\begin{array}{l}
p=\frac{-1+\frac{1}{c}+0.1 k_{1}}{\frac{1}{3}+\frac{0.06 k_{1}}{k_{2}^{2}}}, \\
g=\frac{0.5}{c\left(\frac{1}{3}+\frac{0.06 k_{1}}{k_{2}^{2}}\right)},
\end{array}\right.
$$

which are both always positive for $c<1, k_{1} \geq 0$ and $k_{2}>0$. Hence, $\Delta$ in Eq. (10) will always be positive for $c<1, k_{1} \geq 0$ and $k_{2}>0$, ensuring the uniqueness of the fixed point $\left(v_{e}, w_{e}, \phi_{e}\right)$ in Eq. (11).

With initial conditions at the unique fixed point $\left[v_{e}\left(c, k_{1}, k_{2}\right), w_{e}\left(c, k_{1}, k_{2}\right), \phi_{e}\left(c, k_{1}, k_{2}\right)\right]$, we numerically computed a codimension-one and codimensiontwo bifurcations, showing the excitable and oscillatory regimes of the memristive neuron with the respect to the parameter $c$ in Fig. 2a and the magnetic gain parameters $k_{1}$ and $k_{2}$ in Fig. 2b, respectively.

The bifurcation diagram in Fig. 2a shows a nonzero inter-spike interval (ISI) for $0 \leq c<c_{h}$, where $c_{h}=$ 0.875 is the super-critical Hopf bifurcation threshold. For $c \geq c_{h}$, there is no spiking, i.e., $I S I=0$, indicating that the neuron is in an excitable regime at $k_{1}=0.1$ and $k_{2}=0.1$. However, it is well-known that variations in these magnetic gain parameters can significantly affect the dynamical response of the neuron [76], thereby switching the neuron's dynamics from an excitable to an oscillatory regime and vice versa, even when $c>c_{h}$. Hence, it is important to determine the range of values of $k_{1}$ and $k_{2}$ in which the neuron will
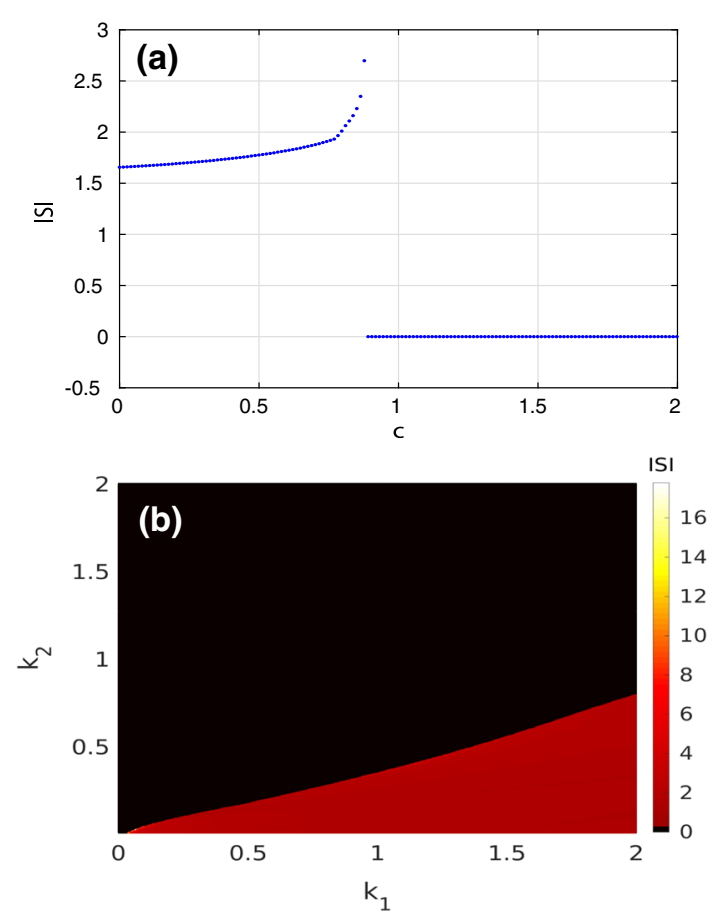

Fig. 2 Panel (a): Bifurcation diagram with respect to parameter $c$, showing the oscillatory (ISI $>0$ ) and excitable (ISI $=0$ ) regimes in $c<c_{h}=0.875$ and $c \geq c_{h}$, respectively, with $k_{1}=0.1$ and $k_{2}=0.1$. Panel (b): Color-coded ISI for a twoparameter space bifurcation diagram with respect to $k_{1}$ and $k_{2}$ at $c=0.95>c_{h}$, showing the oscillatory regime in red and yellow where ISI $>0$ and the excitable regime in dark where ISI $=0$. In both panels, the other parameter values are fixed at: $a=0.1$, $b=0.02, d=0.5$, and $\varepsilon=0.001$

remain in the excitable regime for a particular value of $c$, chosen such that $c_{h}<c<1$.

Figure $2 \mathrm{~b}$ shows, for $c=0.95>c_{h}=0.875$ (i.e., $c$ is far enough from the bifurcation threshold and also less than one so that the stable fixed point is unique), a two-parameter space bifurcation diagram with respect to $k_{1}$ and $k_{2}$. We also note that $k_{2}$ starts at a nonzero value, i.e., at $k_{2}=0.01$, to ensure that our fixed point in Eq. (11) is unique. The color-coded ISI shows the oscillatory regime in red and yellow where ISI $>0$. The yellow region corresponds to few points around the origin of the $\left(k_{1}, k_{2}\right)$ plane, where ISI takes relatively large values. For example, at $k_{1}=0.0361$ and $k_{2}=0.01$ we have ISI $=10.16$, and at $k_{1}=0.0643$ and $k_{2}=0.03$, ISI takes its largest value, i.e., ISI $=17.78$. The dark region (where ISI $=0$ ) corresponds to the excitable regime, with the deterministic model in Eq. (1) consisting of unique and stable fixed point given by Eq. (11). 
Therefore, throughout this paper, we will investigate the mechanism of SISR when the neuron is in the excitable regime defined by: $c=0.95, k_{1} \in[0.0,2.0]$, $k_{2} \in[1.0,2.0], a=0.1, b=0.02, d=0.5$, and $\varepsilon=0.001 \ll 1$.

\section{The asymptotic matching of timescales and SISR}

Now we consider Eq. (1) such that its deterministic version is in the excitable regime, defined by the parameters intervals and values above. To understand how noise can induced a regular escape of trajectories from the basin of attraction of the stable fixed point, leading to the emergence of a coherent spike train, we transform Eq. (1) from the slow timescale $\tau$ to the fast timescale $t$ to obtain Eq. (14) using the relation $\varepsilon:=\tau / t$ or more precisely, $d \tau=\varepsilon d t$ [71]. Under this timescale transformation, the noise term is re-scaled according to the scaling law of Lévy motion. That is, if $L_{\tau}$ is a Lévy motion, then for every $\lambda>0, \lambda^{-\frac{1}{\alpha}} L_{\lambda \tau}$ is also a Lévy motion (i.e., they have the same distribution). Furthermore, we consider the standard form of the Lévy noise, i.e., $L^{\alpha, \beta}(\tau ; \sigma, 0)=\sigma \hat{L}^{\alpha, \beta}(\tau ; 1,0)$, where the scale parameter $\sigma$ clearly represents the noise intensity. We note that because of this scaling law, the term $1 / \sqrt[\alpha]{\varepsilon}$ was introduced in the noise term in Eq. (1) to guarantee that in Eq. (14), the noise intensity, $\sigma$, measures the relative strength of the noise term compared to the deterministic term $f_{1}\left(v_{t}, w_{t}, \phi_{t}\right)$ irrespective of the value of $\varepsilon$.

$$
\left\{\begin{array}{l}
d v_{t}=f_{1}\left(v_{t}, w_{t}, \phi_{t}\right) d t+\sigma d \hat{L}_{t}^{\alpha, \beta}, \\
d w_{t}=\varepsilon f_{2}\left(v_{t}, w_{t}, \phi_{t}\right) d t \\
d \phi_{t}=\varepsilon f_{3}\left(v_{t}, w_{t}, \phi_{t}\right) d t
\end{array}\right.
$$

In the adiabatic limit $\varepsilon \rightarrow 0$, the timescale separation between $v_{t}$ and the two other variables $w_{t}$ and $\phi_{t}$ become very large. This indicates that $w_{t}$ and $\phi_{t}$ are frozen on the $O(1)$ fast timescale. Hence, Eq. (14) is approximated by Eq. (15)

$$
\left\{\begin{array}{l}
d v_{t}=-U_{k_{1}}^{\prime}\left(v_{t}\right) d t+\sigma d \hat{L}_{t}^{\alpha, \beta} \\
d w_{t}=0 \\
d \phi_{t}=0
\end{array}\right.
$$

where $U_{k_{1}}^{\prime}\left(v_{t}\right)$ is the derivative of the potential

$U_{k_{1}}(v)=\frac{1}{12} v^{4}-\frac{1-k_{1} \rho(\phi)}{2} v^{2}+w v$,

with respect $v . U_{k_{1}}(v)$ is the double-well potential with the constant solutions of the last two equations in Eq. (15) given by $w$ and $\phi$, respectively. This potential has, respectively, a left local minimum, a saddle, and a right local minimum at

$$
\left\{\begin{array}{l}
v_{l}=2 \sqrt{-\frac{P}{3}} \cos \left(\frac{1}{3} \arccos \left(\frac{3 Q}{2 P} \sqrt{\frac{-3}{P}}\right)+\frac{2 \pi}{3}\right), \\
v_{m}=2 \sqrt{-\frac{P}{3}} \cos \left(\frac{1}{3} \arccos \left(\frac{3 Q}{2 P} \sqrt{\frac{-3}{P}}\right)-\frac{2 \pi}{3}\right), \\
v_{r}=2 \sqrt{-\frac{P}{3}} \cos \left(\frac{1}{3} \arccos \left(\frac{3 Q}{2 P} \sqrt{\frac{-3}{P}}\right)\right),
\end{array}\right.
$$

where $P=3\left[k_{1} \rho(\phi)-1\right]$ and $Q=3 w$, see Fig. 3 .

It was shown in [91,92] that for barrier crossing phenomena driven by Lévy white noise in the double-well potential, the mean exit time from one of the wells increases as a power $\sigma^{-\alpha}$ of the noise intensity $\sigma$ with $\sigma \rightarrow 0$, and not exponentially as with the Gaussian white noise in the limit $\sigma \rightarrow 0[33,93,94]$. By applying the general results presented in [92] to our particular case, we calculated for the double-well potential in Eq. (16), the mean exit times of the Lévy process as:

$$
\left\{\begin{array}{l}
\mathbb{E} T_{\text {exit }}\left(v_{l} \rightarrow v_{r}\right) \approx \frac{\alpha\left|v_{l}\right|^{\alpha}}{\sigma^{\alpha}}, \text { as } \sigma \rightarrow 0 \\
\mathbb{E} T_{\text {exit }}\left(v_{r} \rightarrow v_{l}\right) \approx \frac{\alpha v_{r}^{\alpha}}{\sigma^{\alpha}}, \text { as } \sigma \rightarrow 0 .
\end{array}\right.
$$

We note that the mean exit times in Eq. (18) depend on the location of the local minima $v_{l}$ and $v_{r}$. We further recall that the mean exit times of the processes driven by $\alpha$-stable noise are much shorter than those of Gaussian processes because of the presence of large jumps which occur with probability polynomially small in $\sigma$ [92].

On the other hand, the mean exit times of a Gaussian process follow Kramers' law [33,93], with escape events occurring with exponentially small probabilities, and are given by:

$$
\left\{\begin{array}{l}
\mathbb{E} T_{\text {exit }}\left(v_{l} \rightarrow v_{r}\right) \approx \exp \left(\frac{2 \Delta U_{-}}{\sigma^{2}}\right), \text { as } \sigma \rightarrow 0 \\
\mathbb{E} T_{\text {exit }}\left(v_{r} \rightarrow v_{l}\right) \approx \exp \left(\frac{2 \Delta U_{+}}{\sigma^{2}}\right), \text { as } \sigma \rightarrow 0
\end{array}\right.
$$

where $\Delta U_{ \pm}$are the energy barrier functions that depend, technically, on $w$ and $\phi$. The asymmetry of the potential in Eq. (16) is controlled only by the sign of the coefficient of the linear term, i.e., the sign of $w$. While the depths of the wells $\Delta U_{ \pm}$are controlled by the value of $w$ and more significantly, by the term $k_{1} \rho(\phi) / 2$. But in the limit as $\varepsilon \rightarrow 0$ in Eq. (14), the magnetic variable $\phi$ becomes almost constant and only the magnetic gain parameter $k_{1}$ now significantly changes the depths of 

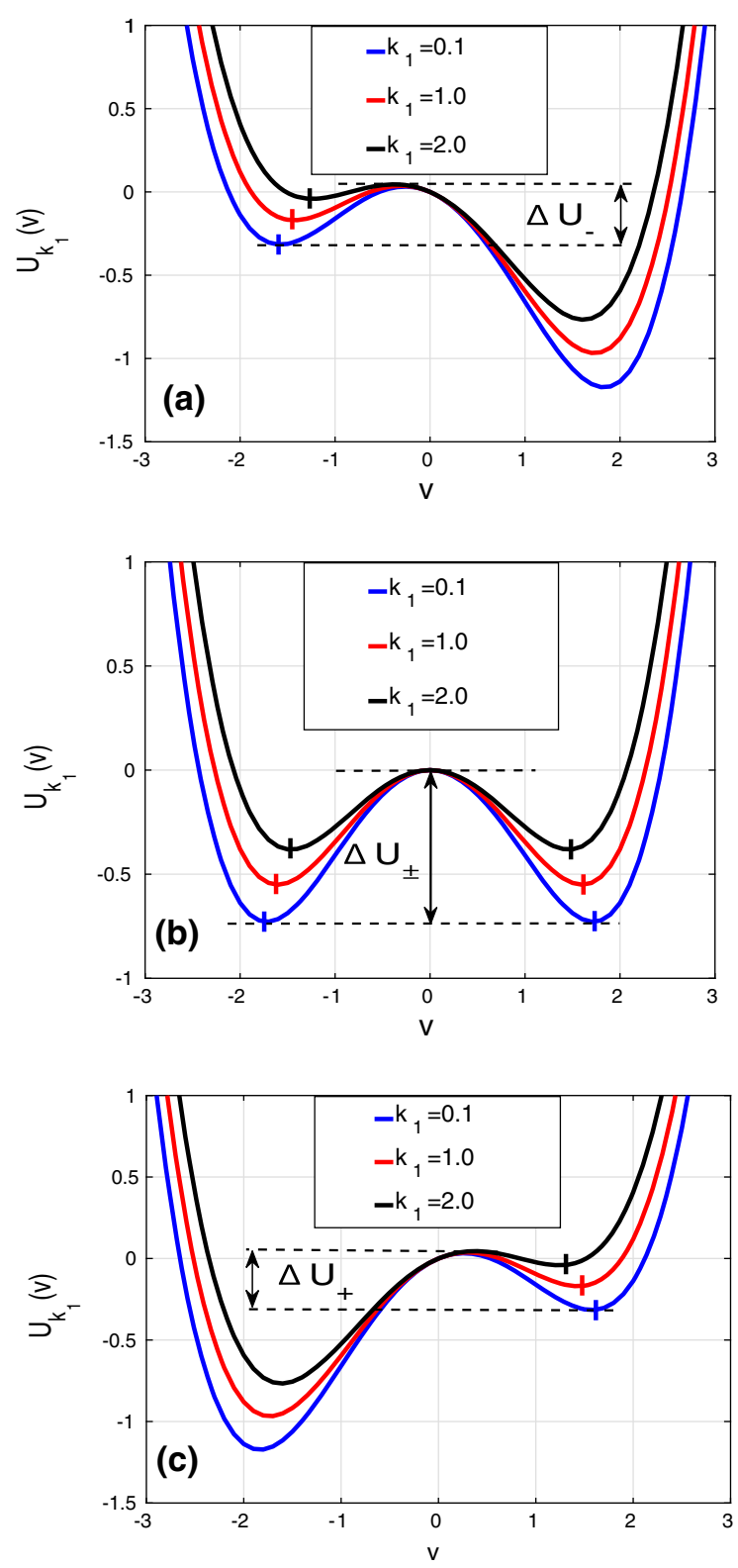

Fig. 3 Variations of the potential $U_{k_{1}}(v)$ given in Eq. (16). The energy barriers $\Delta U_{ \pm}$are indicated in the asymmetric cases in (a) $(w<0)$ and in (c) $(w>0)$, and in the symmetric case in (b) $(w=0)$. The band widths of the wells are given by the distances between the minima located at $v_{l}$ and $v_{r}$ (short vertical bars) and the saddle point located at $v=v_{m}=0$. The stronger the magnetic gain parameter $k_{1}$, the shallower the energy barriers $\triangle U_{ \pm}$and the shorter the band widths. In (a), $w=-0.25$, in (c) $w=0.25$, and in all panels $\phi=0.85$

the potential wells $\triangle U_{ \pm}$. So we can drop the $\phi$ dependence in the energy barrier functions and write them as:

$$
\left\{\begin{array}{l}
\triangle U_{-}(w):=U_{k_{1}}\left(v_{m}\right)-U_{k_{1}}\left(v_{l}\right) \\
\triangle U_{+}(w):=U_{k_{1}}\left(v_{m}\right)-U_{k_{1}}\left(v_{r}\right) .
\end{array}\right.
$$

Thus, in the Gaussian case, the trajectories surmount the potential barriers $\Delta U_{ \pm}$, such that the mean exit times depend exponentially on the depth of the potential wells.

We notice in Fig. 3 that the depths of these barriers are inversely proportional to the strength of the magnetic gain parameter $k_{1}$. Thus, a stronger magnetic flux due to a larger value of $k_{1}$ should, on average, reduce the duration of the mean exit times of the trajectory perturbed by Gaussian noise, contributing to an increase in the spiking frequency.

On the other hand, we also notice that the positions of the minima (at $v_{l}$ and $v_{r}$, indicated by the short vertical bars in Fig. 3) with respect to the fixed saddle (at $v_{m}=0.0$ ) change with $k_{1}$. We observe that the stronger magnetic flux $k_{1}$, the smaller the distances of $v_{l}$ or $v_{r}$ from 0.0 , which in turn shortens, on average, the duration of the mean exit times of the trajectory perturbed by Lévy noise, contributing to an increase in the spiking frequency.

From Eq. (1), the deterministic timescale at which trajectories move on the stable parts of the 2-dimensional cubic nullcline of the model, given by $w(v, \phi)=$ $-\frac{v^{3}}{3}+\left(1-k_{1} \rho(\phi)\right) v$ (not shown), is $\varepsilon^{-1}$ [33]. When there is no noise $(\sigma=0)$, the neuron is in the excitable regime and as $\varepsilon \rightarrow 0$, trajectories tend to spend a lot of time moving adiabatically along the stable parts of the 2D cubic nullcline, toward the unique stable fixed point at $\left(v_{e}, w_{e}, \phi_{e}\right)$ given by Eq. (11), where it stops and stay until a new perturbation (e.g., a random process) is invoked.

When the noise is switched on (i.e., $\sigma \neq 0$ ), it may kick a trajectory, which is moving quasideterministically at a timescale of $\varepsilon^{-1}$ along one stable branch of the 2D cubic nullcline, to another branch and then back. This corresponds to jumps out of the left and right potential wells, thereby causing a spike — an oscillation. Depending on the type of noise perturbing the neuron, an escape from left to right (right to left) occurs at the stochastic timescale of $\mathbb{E} T_{\text {exit }}$ given by the first (second) equation of Eq. (18) for the Lévy process or Eq. (19) for the Gaussian process.

It has been shown that the occurrence of SISR crucially depends on the neuron's ability to asymptotically match, with probability close to unity, the deterministic timescale $\varepsilon^{-1}$ (i.e., timescale at which a trajectory 
moves along the stable parts of the 2D cubic nullcline) and the stochastic timescale $\mathbb{E} T_{\text {exit }}$ (i.e., the timescale at which this trajectory escapes from the stable parts of this nullcline) at the unique exit points $w_{-}$and $w_{+}$located, respectively, on the left and right stable branches of the 2D cubic nullcline [25,27-35].

If the deterministic timescale is shorter than the stochastic timescales (i.e., $\varepsilon^{-1}<\mathbb{E} T_{\text {exit }}$ ), then the trajectory has no time to escape from the left and right stable branches of the cubic nullcline which, respectively, correspond to the left and right wells of the potential $U_{k_{1}}(v)$. Because the neuron is in an excitable regime, the trajectory gets trapped in the left well of the potential (i.e., on the left stable branch of the cubic nullcline on which the unique stable fixed point is located) for too long. In this scenario, a spike is a rare event and this could destroy the coherence of the spiking, especially for short time intervals.

On the other hand, if the deterministic timescale is longer than the stochastic timescales (i.e., $\varepsilon^{-1}>$ $\left.\mathbb{E} T_{\text {exit }}\right)$, then the trajectory frequently escape from the potential wells (i.e., the stable branches of the cubic nullcline). In this scenario, spiking is frequent (i.e., not rare) but incoherent because the trajectory escapes at several different points on each of the stable branches of the cubic nullcline.

Interestingly, if at specific and unique points $w_{-}$ and $w_{+}$on the left and right stable branches of the cubic nullcline, respectively, the deterministic timescale matches the stochastic timescales (i.e., $\varepsilon^{-1}=$ $\left.\mathbb{E} T_{\text {exit }}\right)$, frequent and coherent spiking emerges SISR occurs. The uniqueness of the exit points $w_{-}$ and $w_{+}$can only be guaranteed by the monotonicity of the minima $v_{l}(w)$ and $v_{r}(w)$ in the case of Lévy noise (see Eq. 21) and the barrier functions $\triangle U_{-}(w)$ and $\triangle U_{+}(w)$ in the case of Gaussian noise (see Eq. 22).

In Fig. 4, we show the graphs of the functions $\left|v_{l}\right|$, $v_{r}, \Delta U_{-}$, and $\triangle U_{+}$with respect to $w \in\left[-\frac{2}{3}, \frac{2}{3}\right]$, where the lower and upper bounds of this interval correspond to the $w$-coordinate of the local minimum and maximum of the cubic nullcline, respectively. Here, we see that these functions are all monotone with respect to $w \in\left[-\frac{2}{3}, \frac{2}{3}\right]$. Hence, frequent and coherent spiking would occur if we match the deterministic and stochastic timescales only at $w_{-}$on the left stable branch and at $w_{+}$on the right stable branch of the cubic nullcline, that is:

$$
\frac{\alpha\left|v_{l}\left(w_{-}\right)\right|^{\alpha}}{\sigma^{\alpha}}=\frac{1}{\varepsilon}=\frac{\alpha v_{r}\left(w_{+}\right)^{\alpha}}{\sigma^{\alpha}},
$$

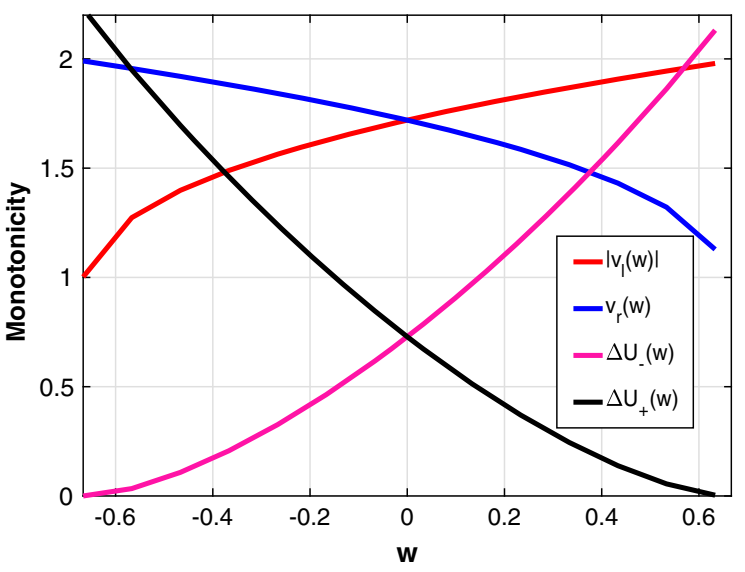

Fig. 4 The graphs of the $\left|v_{l}(w)\right|, v_{r}(w), \Delta U_{-}(w)$, and $\Delta U_{+}(w)$ with respect to $w \in\left[-\frac{2}{3}, \frac{2}{3}\right]$. Their monotonicity ensure the uniqueness of the escape points $w_{-}$and $w_{+}$which satisfy the equations in Eqs. (21 and 22). Parameters are $k_{1}=0.1$ and $\phi=0.85$

for the Lévy process, and

$\exp \left(\frac{2 \Delta U_{-}\left(w_{-}\right)}{\sigma^{2}}\right)=\frac{1}{\varepsilon}=\exp \left(\frac{2 \Delta U_{+}\left(w_{+}\right)}{\sigma^{2}}\right)$,

for the Gaussian process. Therefore, the occurrence of SISR (i.e., frequent and coherent spiking) will depend on the neurons' ability to asymptotically match the timescales by taking the following double scaling limits:

$$
\lim _{(\varepsilon, \sigma) \rightarrow(0,0)}\left[\sigma^{\alpha} \varepsilon^{-1}\right] \rightarrow\left\{\begin{array}{l}
\alpha\left|v_{l}\left(w_{-}\right)\right|^{\alpha} \\
\alpha v_{r}\left(w_{+}\right)^{\alpha}
\end{array}\right.
$$

for the Lévy process, and

$$
\lim _{(\varepsilon, \sigma) \rightarrow(0,0)}\left[\frac{\sigma^{2} \ln \left(\varepsilon^{-1}\right)}{2}\right] \rightarrow\left\{\begin{array}{l}
\Delta U_{-}\left(w_{-}\right) \\
\Delta U_{+}\left(w_{+}\right)
\end{array}\right.
$$

for the Gaussian process [27,33].

Due to the anomalous long jumps of a trajectory perturbed by a Lévy process [92,95-97], this trajectory does not necessarily have to hit the saddle point at $v_{m}$ before escaping from the stable branches of the $2 \mathrm{D}$ cubic nullcline. Hence, escapes may instantaneously occur even with a very weak noise intensity. This means that the "frequent spiking" requirement of SISR can be easily achieved by a Lévy process, even with a very weak noise intensity. However, the "coherent spiking" requirement of SISR can only be guaranteed by the asymptotic scaling limits given in Eq. (23).

In the Gaussian case, a trajectory can only escape from a potential well after hitting the boundary at the 
saddle point at $v_{m}$. Therefore, the "frequent spiking" requirement of SISR needs that the noise intensity is not too weak (otherwise, we get a Poissonian spike traina rare spiking event which could destroy the coherence of the spiking [33]). Moreover, we observe that the stochastic timescales of the Gaussian noise in Eq. (19) depend on the energy barrier functions $\triangle U_{ \pm}$. If these barriers are too deep (i.e., $\Delta U_{ \pm} \rightarrow \infty$ ), then weak noise intensities cannot provoke escapes (at least frequently), and the trajectory will remain strapped inside a potential well. Thus, the noise has be to weak (so that the mean exit times satisfy Eq. 19), but strong enough to able to invoke some spiking. If this Gaussian noise is strong enough to invoke spiking, then the "coherent spiking" requirement of SISR can only be guaranteed by the asymptotic scaling limits given by Eq. (24). Thus, for Lévy noise, we expect SISR to occur even at very weak noise intensities. But for Gaussian noise, we expect SISR to occur at a comparatively larger intensity.

To answer the three main questions we are interested in (see the introduction section), we will set the memristive neuron in the excitable regime by choosing $c=0.95, a=0.1, b=0.02, d=0.5$, and also set location parameter of the standardized Lévy process at $\mu=0.0$. We chose a sufficiently small timescale separation parameter, i.e., $\varepsilon=0.001 \ll 1$, weak noise intensities, i.e., $0<\sigma \ll 1$, and then numerically search for the combined values of $k_{1} \in[0.0,2.0]$, $k_{2} \in[1.0,2.0], \alpha \in(0,2]$, and $\beta \in[-1,1]$ for which the scaling limit conditions in Eqs. (23 and 24) are satisfied (or at least to some degree) or not.

\section{Numerical results and discussion}

To measure the degree of SISR (i.e., the degree to which Eqs. (23 and 24) are satisfied), we use the coefficient of variation $(\mathrm{CV})$, an important statistical measure based on the time intervals between spikes [15]. From a neurobiological point of view, $\mathrm{CV}$ is more important than other measures (e.g., power spectral density and autocorrelation function) because it is related to the timing precision of information processing in neural systems [98]. CV uses the inter-spike intervals (ISIs) where the $k$ th interval is the difference between two consecutive spike times $t^{k}$ and $t^{k+1}$ of the neuron, and is defined as:
$\mathrm{CV}=\frac{\sqrt{\left\langle\mathrm{ISI}^{2}\right\rangle-\langle\mathrm{ISI}\rangle^{2}}}{\langle\mathrm{ISI}\rangle}$,

where $\langle\mathrm{ISI}\rangle$ and $\left\langle\mathrm{ISI}^{2}\right\rangle$ represent the mean and the mean squared ISIs, respectively. When $\mathrm{CV}=1$, we have Poissonian spike train (i.e., rare and incoherent spiking), and when $\mathrm{CV}>1$ we have a point process that is even more variable than a Poisson process, i.e., a point process whose standard deviation is larger than the mean [99-101].

In both these cases, the degree of SISR is quite low as the double limits in the left-hand sides of Eqs. (23 and 24) fail to converge toward the corresponding values on the right-hand sides. The degree of SISR becomes higher with $\mathrm{CV} \rightarrow 0$ as the double limits in the lefthand sides of Eqs. (23 and 24) also converge toward the corresponding values on the right-hand sides. When $\mathrm{CV}=0$, the double limits in the left-hand sides of Eqs. (23 and 24) should be exactly equal to the corresponding values on the right-hand sides. In this case, we will have perfectly "deterministic" periodic spiking.

For our numerical simulations, we used the fourthorder stochastic Runge-Kutta algorithm employed in $[6,102,103]$ and given in Appendix (A.1). This scheme has been proven in [104] to strongly converge. In should be noted that for general noise, the numerical solution of stochastic differential equations that uses the scheme proposed by Wilkie [105] may not be intact even with additive noise, see also [106]. We numerically integrate Eq. (14) for a very long time interval (i.e., $T=4 \times 10^{7}$ time unit which allows, for the small value of $\varepsilon=$ 0.001 , the collection of sufficiently many ISIs for the statistical estimate).

The time step used in our simulations is $d t=0.01$ (note: smaller time step, i.e., $d t=0.005$ do not show any changes in the results presented but required more lengthy simulation time). The results presented were average over 30 independent realizations for each set of parameter values to warrant appropriate statistical accuracy with respect to the numerical simulations. For each realization, we choose random initial points with a uniform probability in the range of $v(0) \in(-2,2)$, $w(0) \in(-2 / 3,2 / 3)$, and $\phi(0) \in(-2,2)$. The result presented is robust to the choice of initial conditions and this is not surprising because the system has been configured such that it has a unique stable fixed point (i.e, there is no multi-stability, in which case the choice of initial conditions does not affect the results presented). 
Fig. 5 The variation of CV with the noise intensity $\sigma$ with Lévy noise ( $\alpha=0.1$, $\beta=0.0)$ in (a) and Gaussian noise $(\alpha=2.0$, $\beta=0.0)$ in (b). Time series during SISR induced by the Lévy noise in (c) with $\sigma=0.04$ and Gaussian noise in (d) with $\sigma=0.04$. Degree of SISR is higher with Lévy noise than with Gaussian noise for all values of $\sigma . k_{1}=0.1$ and $k_{2}=0.1$
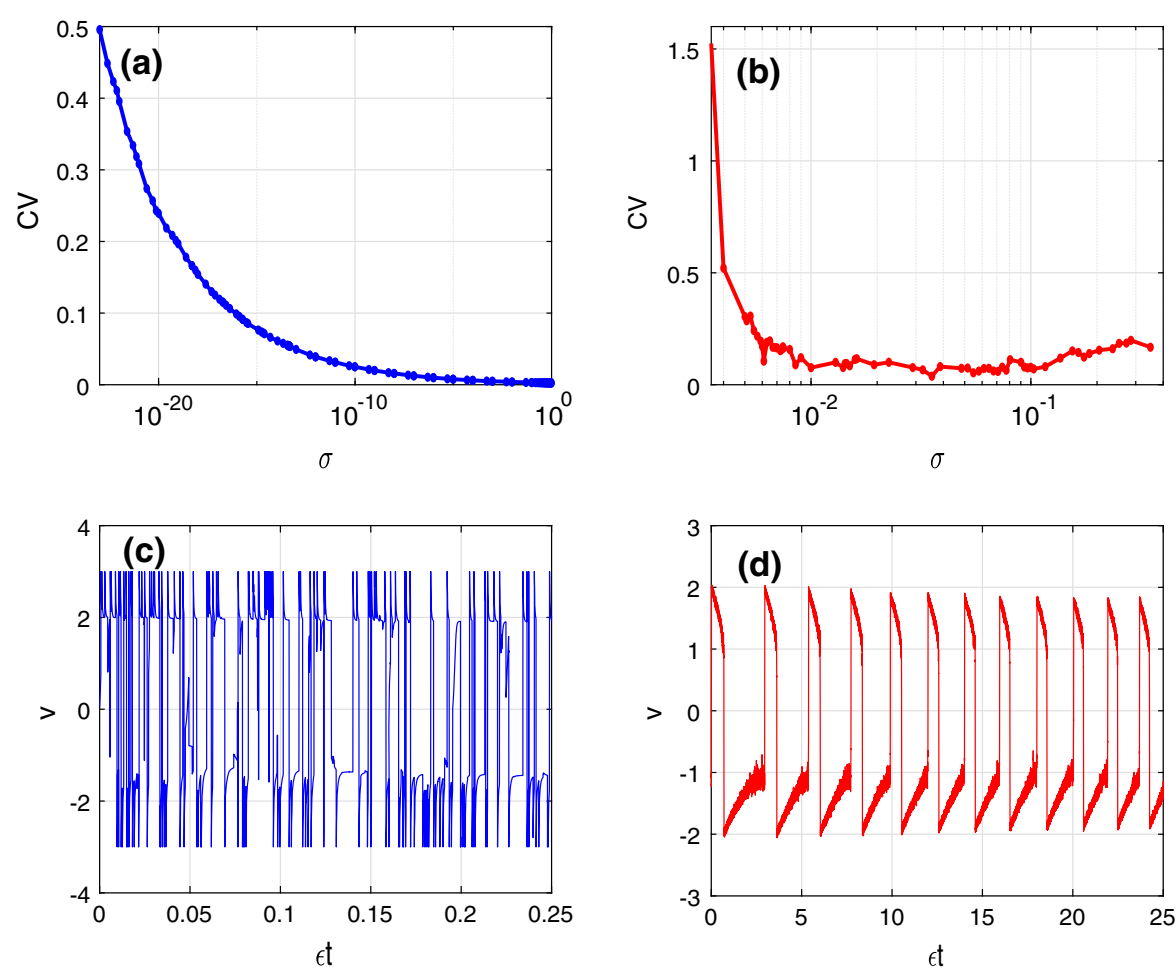

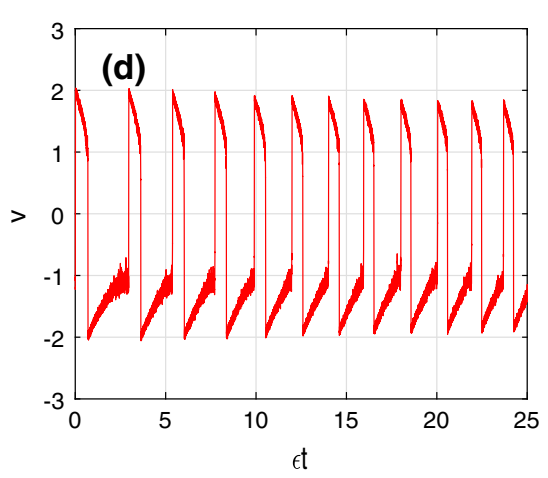

We recall that the continuous jump property of a Gaussian process (with finite variance) forces the trajectories to hit the boundary of a domain before escaping. While with the discontinuous long-jumps of a Lévy process with $\alpha<2$ (with infinite variance), trajectories can rapidly escape to infinity without hitting the boundary. Thus, for our FHN neuron perturbed by a Lévy noise, we might need to wait for a long time for a trajectory which had exhibited a long-jump to come back to the vicinity of the stable fixed point, if there is no compulsory truncation. It is important to note that these long waiting times can significantly affect the ISIs. Hence, because the CV (used to characterised the degree of SISR) depends (only) on the ISIs, the numerical results obtained would be sensitive to the choice of the truncation threshold. Considering the physical and computer saturation effects, a suitable truncation scheme should, therefore, be adoptable. In our simulations, we use the truncation threshold $v=3.0 \times$ $\operatorname{sign}(v)$ whenever $|v|>3.0$. This is a well-known truncation scheme for $\alpha$-stable noises employed in many relevant references [107-109].

To avoid the long waiting times to which $\mathrm{CV}$ is sensitive to, we decided to use the truncation threshold above. We note that the threshold values (i.e., $v=-3$ and $v=3$ ) are, respectively, below and above, but also sufficiently close to the extreme values ( $v=-2$ and $v=2$, see Fig. 5d) of the relaxation oscillations of the underlining deterministic FHN model. A value of, for example, $v=1000$ is not physiological for the FHN model. Thus, the truncation threshold used not only ensures that the simulated trajectories do not escape to infinity (thereby avoiding the long waiting times) but also ensures that the trajectories do not go too far below and above the extreme values of the relaxation oscillation, which are in fact the physiologically acceptable extreme values for the model. In the presence of noise, the random trajectories may then oscillate with slightly bigger amplitudes compared to that of the deterministic relaxation oscillation. Thus, the truncation scheme used gives room for these fluctuations to be taken into account without any significant effect on the waiting times that arise due to the long-jumps. These makes the truncation threshold $v=3 \times \operatorname{sign}(v)$ whenever $|v|>3$, a good and natural choice for calculating the $\mathrm{CV}$ values of the FHN model perturbed by a Lévy noise.

Figure 5a and c respectively shows the variation of $\mathrm{CV}$ with the noise intensity $\sigma$ for a very impulsive ( $\alpha=0.1)$ and symmetric $(\beta=0.0)$ Lévy noise and a time series of the coherent spike trains obtained at 
a noise intensity that satisfies Eq. (23). The CV-curve and time series are computed in a weak magnetic flux regime $\left(k_{1}=0.1, k_{2}=0.1\right)$ and show that as long as Eq. (23) is valid, Lévy noise can ( $i$ ) induce a high degree of SISR even at very weak noise intensities (e.g., $\mathrm{CV} \approx 0.075$ at $\sigma=1.0 \times 10^{-15}$ ), and (ii) induce an even higher degree of SISR at relatively larger noise intensities (e.g., $\mathrm{CV}=0.0015$ at $\sigma \approx 0.9$ ). It is worth noting that in Fig. 5a and $\mathrm{c}$ the Lévy noise is very impulsive, i.e., the stability index is very small ( $\alpha=0.1)$, and therefore even at very weak noise intensities (such as $\sigma=1.0 \times 10^{-20}$ ), the long-jumps can still occasionally occur, thereby inducing some spikes whose ISI $s \neq 0$ will contribute to a finite $\mathrm{CV}$ value. But as $\alpha$ increases, the long-jumps become less frequent and of shorter range. Thus, only relatively larger noise intensities can invoke spikes as in Gaussian case in Fig. 5b and d.

In Fig. $5 \mathrm{~b}$ and d, we respectively show the variation of CV with the noise intensity $\sigma$ for Gaussian noise $(\alpha=2.0, \beta=0.0)$ and a time series of the coherent spike train obtained at a noise intensity which satisfies Eq. (24), in the same weak magnetic flux regime $\left(k_{1}=\right.$ $\left.0.1, k_{2}=0.1\right)$. Comparing the degree of SISR induced by a Lévy noise with parameters at $\alpha=0.1$ and $\beta=$ 0.0 to that of Gaussian noise $(\alpha=2.0, \beta=0.0)$, we see that Lévy noise can induce a higher degree of SISR with both extremely weak and weak noise amplitudes. In Fig. $5 \mathrm{~b}$ with Gaussian noise, we have a low (and almost constant) $\mathrm{CV} \approx 0.045$ only in the weak (but not too weak) noise intensities, i.e., for $\sigma \in(0.01,0.1)$.

Figure $6 \mathrm{a}$ and $\mathrm{b}$ shows minimum coefficient of variation $\left(\mathrm{CV}_{\text {min }}\right)$ against the stability index $(\alpha)$ and the skewness $(\beta)$ parameters of the Lévy process in a weak $\left(k_{1}=0.1\right.$ and $\left.k_{2}=0.1\right)$ and in a strong $\left(k_{1}=2.0\right.$ and $\left.k_{2}=1.0\right)$ magnetic flux regime, respectively. In Fig. 6a, a weak magnetic flux regime $\left(k_{1}=0.1\right.$, $k_{2}=0.1$ ), a right-skewed (i.e., $\left.\beta \in(0.0,1.0]\right)$ Lévy process with a low stability index (i.e., $\alpha \in(0.0,0.7])$ can induce a high degree of SISR, as indicated by the very low value of $\mathrm{CV}_{\text {min }} \approx 0.0014$. With higher values of $\alpha$, i.e., for $\alpha \in(1.0,2.0)$ and irrespective of the value of the skewness parameter, i.e., for $\beta \in[-1.0,1.0]$, the degree of SISR is high and almost constant as indicated by the low and almost constant $\mathrm{CV}_{\text {min }} \approx 0.005$. Even though this cannot be clearly seen from the panel, the data show that, at $\alpha=2.0$ and $\beta \in[-1.0,1.0]$ (which includes the Gaussian case at $\beta=0.0$ ), the $\mathrm{CV}_{\text {min }}$ is also low and almost constant at $\mathrm{CV}_{\text {min }} \approx 0.0497$, i.e, almost 10 order of mag- nitude higher than the $\mathrm{CV}_{\text {min }}$ of the Lévy processes in which $\alpha \in(1.0,2)$ and $\beta \in[-1.0,1.0]$. And for $\alpha \in(0.0,1.0]$ and $\beta \in[-1.0,-0.5]$ (i.e., from the bright red, the yellow, and the white regions), the degree of SISR is relatively low, as $\mathrm{CV}_{\text {min }}$ continuously vary in the interval $\mathrm{CV}_{\min } \in[0.125,0.328]$, with the highest value of $\mathrm{CV}_{\text {min }} \approx 0.328$, occurring at $\alpha=0.8$ and $\beta=-1.0$.

In Fig. 6b, with a strong magnetic flux regime $\left(k_{1}=\right.$ $2.0, k_{2}=1.0$ ), the variation in the degree of SISR is qualitatively the same as in Fig. 6a, but the data show that there is a slight quantitative difference in the oder of magnitude of the $\mathrm{CV}_{\text {min }}$ values, and hence in the degree of SISR in both panels. For example, when we have Gaussian noise (i.e., $\alpha=2.0$ and $\beta=0.0$ ), we have a $\mathrm{CV}_{\text {min }} \approx 0.0538$ for weak magnetic flux in Fig. $6 \mathrm{a}$ and $\mathrm{CV}_{\min } \approx 0.0497$ for strong magnetic flux in Fig. 6b. Later, we shall discuss and show more clearly in the $\left(k_{1}, k_{2}\right)$-plane the effects of the magnetic gain parameters on the degree of SISR.

The presence of intermittent intervals of sub-threshold spiking explains the relatively high values of $\mathrm{CV}_{\text {min }} \in$ $[0.125,0.328]$ in the region bounded by $\alpha \in(0.0,1.0]$ and $\beta \in[-1.0,-0.5]$ (i.e., the bright red, yellow, and white regions) in the panels of Fig. 6. Because of these intervals of intermittent sub-threshold spiking (with $v \leq v_{t h}=1.3$, an arbitrarily chosen threshold value), the regularity of the ISIs which is calculated based on the occurrence of supra-threshold spiking (with $v>v_{t h}$ ) is deteriorated. On the other hand, for parameter values in the regions bounded by $\alpha \in(0.0,1.0]$ and $\beta \in(-0.5,1.0]$ (i.e., dark region with $\left.\mathrm{CV}_{\text {min }} \approx 0.0014\right), \alpha \in(1.0,2.0)$ and $\beta \in$ $[-1.0,1.0]$ (i.e., dark region with $\mathrm{CV}_{\text {min }} \approx 0.005$ ), and by $\alpha=2.0$ and $\beta \in[-1.0,1.0]$ (i.e., dark region with $\mathrm{CV}_{\text {min }} \approx 0.0497$ ), the time series contain fewer intermittent intervals of sub-threshold spiking (see, e.g., Fig. 5c), hence the low value of the $\mathrm{CV}_{\min }$ in these regions.

In Fig. 7, we show the variation in the degree of SISR with variations in the strengths of the magnetic gain parameters $k_{1}$ and $k_{2}$ in three specific regions of interest in Fig. 6a: (i) when the degree of SISR is low, i.e., in the white spot with $\alpha=0.7$ and $\beta=-1.0$, (ii) when the degree of SISR is high, i.e., the dark red region with $\alpha=2.0$ and $\beta=0.0$ (i.e., Gaussian), and (iii) when the degree of SISR is very high, i.e., the black region with $\alpha=0.1$ and $\beta=1.0$. We also note that in all the panels of Fig. 7, the magnetic gain parameter $k_{2}$ 

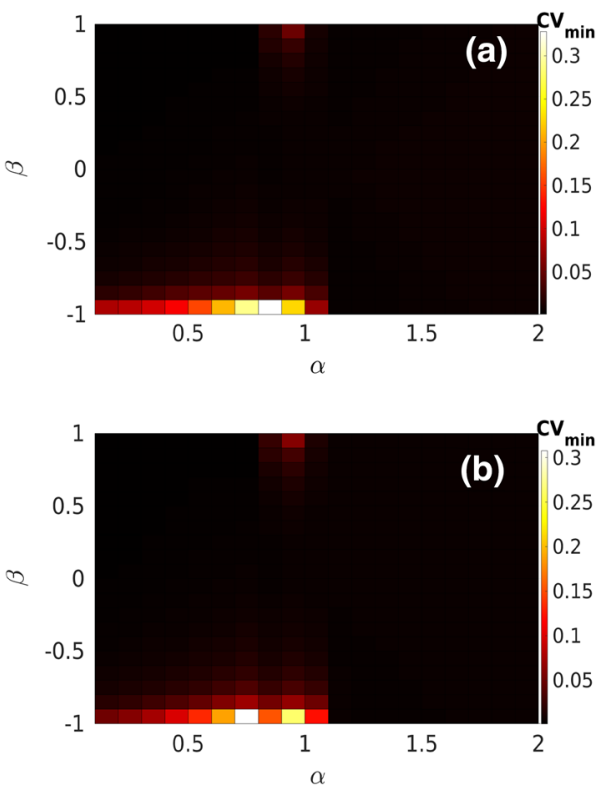

Fig. 6 Variations of the minimum $\mathrm{CV}\left(\mathrm{CV}_{\text {min }}\right)$ with respect to the stability index $(\alpha)$ and the skewness $(\beta)$ parameters with weak $\left(k_{1}=0.1, k_{2}=0.1\right)$ and strong $\left(k_{1}=2.0, k_{2}=1.0\right)$ magnetic gain parameters in (a) and (b), respectively

is restricted to $k_{2} \geq 1.0$, so that the memristive neuron always lies in the excitability region (black region) for all values of $k_{1} \geq 0.0$, as indicated in Fig. $2 b$.

In Fig. 7a, we can now clearly see the effects of the magnetic gain parameters on the degree of SISR when $\alpha=0.7$ and $\beta=-1.0$, corresponding, from Fig. 6a, to the white spot with a relatively large $\mathrm{CV}_{\text {min }} \approx 0.328$. We observe that: the stronger the magnetic gain parameter $k_{1}$ (that bridges the coupling and modulation on the membrane potential $v$ from magnetic field $\phi$ ) and the weaker the parameter $k_{2}$ (that describes the degree of polarization and magnetization by adjusting the saturation of magnetic flux), the higher the degree of SISR. In Fig. 7a, as $k_{1} \rightarrow 2.0$ and $k_{2} \rightarrow 1.0$, the color-coded $\mathrm{CV}_{\text {min }}$ goes from a white region with a relatively high value of $\mathrm{CV}_{\text {min }} \approx 0.69$, via a yellow and a red, to a black region with the lowest $\mathrm{CV}_{\text {min }} \approx 0.30$. Moreover, irrespective of the value of $k_{2}$, when $k_{1}=0.0, \mathrm{CV}_{\text {min }}$ takes the highest value of the panel (i.e., $\mathrm{CV}_{\min } \approx 0.69$ in the white region). Further numerical simulations (not shown) indicated that this behavior is qualitatively the same for many pairs of values of $\alpha \in(0.0,1.0]$ and $\beta \in[-1.0,-0.5]$. This means that the appropriate combination of values of the magnetic gain parameters can significantly improve the degree of SISR induced
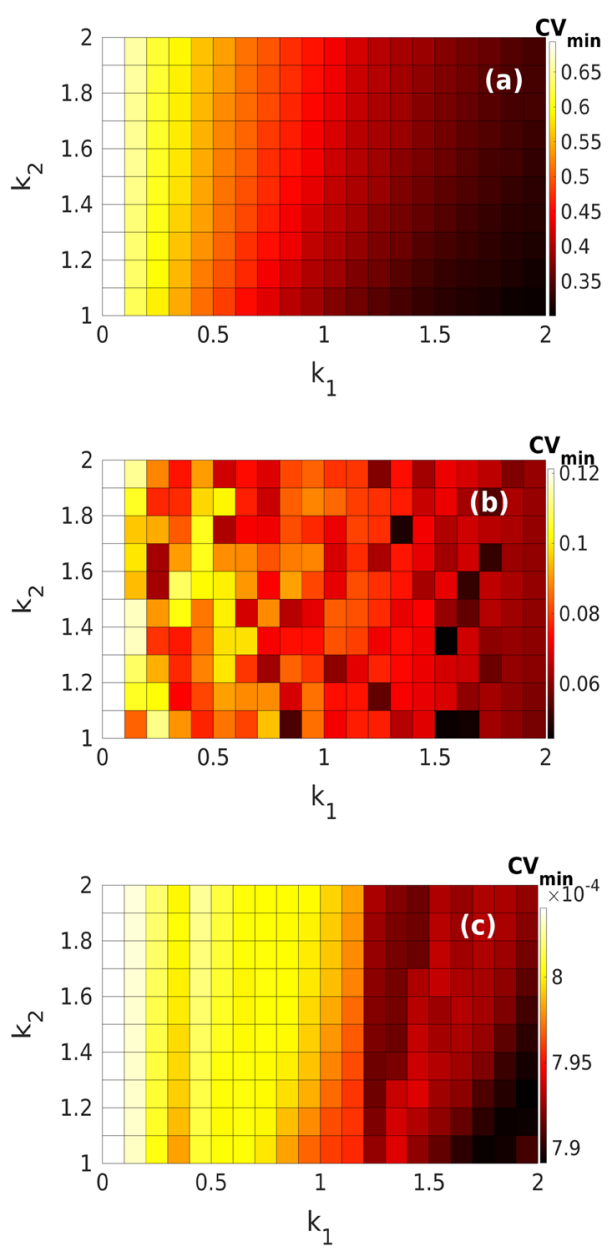

Fig. 7 Variations of the minimum $\mathrm{CV}\left(\mathrm{CV}_{\text {min }}\right)$ with respect to the magnetic gain parameters $k_{1}$ and $k_{2}$ at different values of the stability index and skewness parameters. In all cases, the larger $k_{1}$ is and the smaller $k_{2}$ is, the lower is the value of $\mathrm{CV}_{\min }$, i.e., the higher the degree of SISR. In (a): $\alpha=0.7, \beta=-1.0$; in (b): $\alpha=2.0, \beta=0.0$; and (c): $\alpha=0.1, \beta=1.0$

by Lévy noise when the noise parameters are in intervals $\alpha \in[0.0,1.0]$ and $\beta \in[-1.0,-0.5]$. We shall see later in Fig. 7c that this significant improvement in the degree of SISR depends on intervals in which $\alpha$ and $\beta$ are located.

In Fig. 7b, we have Gaussian noise (i.e., $\alpha=2.0$ and $\beta=0.0$ ) and effects of the magnetic gain parameters are qualitatively the same as in Fig. 7a with a Lévy noise having parameters at $\alpha=0.7$ and $\beta=-1.0$. That is, the weaker $k_{2}$ and the stronger $k_{1}$ become, the lower is $\mathrm{CV}_{\text {min }}$, on average. It is worth noting, by comparing Fig. 7a and $b$, that the degree of SISR induced by Lévy noise (with $\alpha=0.7$ and $\beta=-1.0$ ) is 
lower than that induced by Gaussian noise ( $\alpha=2.0$ and $\beta=0.0)$. Furthermore, the effects of the magnetic gain parameters $k_{1}$ and $k_{2}$ on the degree of SISR is weaker in the Gaussian case. That is, in Fig. $7 \mathrm{~b}, \mathrm{CV}_{\min }$ varies in the interval [0.044, 0.121], compared to [0.30, 0.69] in Fig. 7a. The bigger range in the latter interval indicates the stronger effects of the magnetic gain parameters on the degree of SISR induced by Lévy noise when its parameters lie in the intervals $\alpha \in(0.0,1.0]$ and $\beta \in[-1.0,-0.5]$.

Moreover, it important to note that the degree of SISR in the non-memristive neuron (i.e., when $k_{1}=0$ ) is always lower (poorer) than that in the memristive one. This result is confirmed by comparing $\mathrm{CV}_{\text {min }}$ in the non-memristive FHN neuron perturbed by Gaussian noise — studied in our previous work [33] — to the memristive FHN model studied in the current paper. In the non-memristive case, the lowest $\mathrm{CV}$ value is always at $\mathrm{CV} \approx 0.2$, while in the memristive case, the lowest value gets even smaller, i.e., $\mathrm{CV} \approx 0.044$, especially as $k_{1} \rightarrow 2$ and $k_{2} \rightarrow 1$.

In Fig. 7c, we have a Lévy noise with $\alpha=0.1$ and $\beta=1.0$, which corresponds to a black region (i.e., with a high degree of SISR) in Fig. 6a. In this case, just as in Fig. $7 \mathrm{a}$, as $k_{1} \rightarrow 2$ and $k_{2} \rightarrow 1$, the higher the degree of SISR. However, the magnetic gain parameters $\left(k_{1}\right.$ and $k_{2}$ ) have weaker effects on the high degree of SISR compared to when the Lévy process is very impulsive, as for example, in Fig. 7a. In Fig. 7c, the degree of SISR remains very high with a $\mathrm{CV}_{\min }$ varying within an extremely thin interval of [0.000789, 0.000804], for all values of $k_{1}$ and $k_{2}$. In this case, the Lévy process with $\alpha=0.1$ and $\beta=1.0$ induces a higher degree of SISR than the Gaussian process, in contrast to a Lévy process with $\alpha=0.7$ and $\beta=-1.0$.

In the adiabatic limit $\varepsilon \rightarrow 0$, the fact that stronger magnetic flux $k_{1}$ can significantly improve the degree of SISR with a Gaussian or a Lévy process can theoretically be explained in term of the potential landscapes in Fig. 3 and the mean exit times given by Eqs. (18 and 19). In the Gaussian case, we see (from Eqs. 16 and 20) that $\Delta U_{ \pm}(w)$ depends explicitly on the memristive parameter $k_{1}$ and implicitly on the memristive parameter $k_{2}$ from the term $\rho(\phi)$ in Eq. (16), where $\phi$ in turn depends on $k_{2}$ from the function $f_{3}$ in Eq. (2). The mean exit times depend exponentially on the energy barrier functions $\Delta U_{ \pm}$(see Eq. (19)) which should not be too deep (i.e., $\Delta U_{ \pm}(w) \rightarrow 0$ ), so that weaker noise intensities $(\sigma \rightarrow 0)$ can be sufficient to provoke jumps (spikes) from one potential well to another. So as $k_{1} \rightarrow 2$ (i.e., becomes stronger), $\Delta U_{ \pm} \rightarrow 0$ (i.e., become shallower, see Fig. 3), and the more easily weak noise intensities can provoke frequent spikes. And if this frequent spiking is combined with the scaling limits in Eq. (24), the degree of SISR gets higher (i.e., $\mathrm{CV}_{\text {min }} \rightarrow 0$ ). But when $k_{2}$ increases, it has the opposite effect on $\Delta U_{ \pm}(w)$ through their implicit (non-trivial) dependence on $k_{2}$ via $\phi$ in the term $\rho(\phi)$ in potential in Eq. (16). Therefore, small noise amplitudes can no longer frequently kick trajectory over these barriers, and thus the degree of SISR decreases with increase in $k_{2}$.

In the Lévy cases, a similar theoretical explanation applies but in terms of the locations of the minima $v_{l}$ and $v_{r}$ from the saddle point at $v=v_{m}=0.0$. We observe that the mean exit times in Eq. (18) depend on the location of the minima $v_{l}$ and $v_{r}$ and hence, also on band widths of the wells (i.e., the distances from the minima $v=v_{l}$ and $v=v_{r}$ of the wells to the saddle point $v=v_{m}=0$, (see Fig. 3 which shows a reduction in the distance between the short vertical bars all located at these minima, and the point $v=0$, as $k_{1}$ increases). The shorter these band widths are (i.e., the closer $v_{l}$ and $v_{r}$ are to $v_{m}=0$ ), the shorter the mean exit times given in Eq. (18). Thus, weak noise intensities can more easily provoke frequent jumps (spikes) from one potential well to another. When this frequent spiking is combined with the scaling limits in Eq. (23), the degree of SISR gets higher. On the other hand, $k_{2}$ (on which the minima $v_{l}(w)$ and $v_{r}(w)$ implicitly depend via the term $\rho(\phi)$ in the expression of $P$ in Eq. 17) has the opposite effect on these bandwidths compared $k_{1}$ and consequently on the degree of SISR.

However, when the Lévy noise becomes impulsive (i.e., as $\alpha \rightarrow 0$, with a variance that tends to infinity, see Fig. 1 and also [92]), the anomalous instantaneous long jumps of trajectories becomes significant. In this case, the band widths which are controlled by magnetic gain parameter $k_{1}$ do not longer have significant effects on the mean exit times. Thus, as $\alpha \rightarrow 0$, the variation in the magnetic gain parameters should also not have too much effects on the high degree of SISR, as long as Eq. (23) is satisfied. This is what we observe in Fig. 7c with $\alpha=0.1$ and $\beta=1.0$.

Nevertheless, this inability to significantly change the degree of SISR when $\alpha \in(0.0,1.0]$, depends also on the skewness of the Lévy noise. If the noise is left-skewed (as e.g., in Fig. 7a with, in particular 
$\beta=-1.0$ ), then the left potential well (i.e., the left stable branch of the cubic nullcline on which the unique stable fixed point is located) is favoured compared to the right well (i.e., the right stable branch). This results into trajectories staying a bit longer in this left well, provoking these intermittent intervals of sub-threshold spiking which destroys the regularity of the ISIs. In this left-skewed case, the magnetic gain parameters have significant effect on the degree of SISR as we saw in Fig. 7a.

\section{Summary and conclusions}

In this paper, we investigated and compared the mechanism of SISR induced by Lévy white noise and Gaussian white noise in a memristive FHN neuron. We showed that depending on the parameter values $(\alpha \in$ $(0,2)$ and $\beta \in[-1,1])$ of the Lévy noise, the neuron could exhibit a very high degree of SISR with a minimum coefficient of variation as low as 0.000789 , compared to 0.044 in the case of Gaussian noise. However, the degree of SISR induced by a Lévy noise is not always higher than that induced by the Gaussian noise. In particular, in the intervals $\alpha \in(0.0,1.0]$ and $\beta \in[-1.0,-0.5]$, the Lévy processes induce a lower degree of SISR (with $\mathrm{CV}_{\text {min }} \in[0.125,0.328]$ ) than the Gaussian process with $\mathrm{CV}_{\text {min }} \approx 0.0497$.

It is shown that, the stronger magnetic gain parameter $k_{1}$ (i.e., the parameter that bridges the coupling and modulation on membrane potential $v$ from magnetic field $\phi$ ) and the weaker $k_{2}$ (i.e., the parameter that controls the degree of polarization and magnetization by adjusting the saturation of magnetic field $\phi$ ) are, the higher the degree of SISR for both Lévy and Gaussian processes. However, in the Lévy case, this combined effect of the magnetic gain parameters on the degree of SISR becomes less significant when the process becomes more impulsive (i.e., as $\alpha \rightarrow 0$ ) and rightskewed (with $\beta \rightarrow 1$ ). Moreover, it has been shown, for both types of noises, that the degree of SISR in the memristive neuron (i.e., when $k_{1} \neq 0$ and $k_{2} \neq 0$ ) is always higher than the degree in the non-memristive neuron (i.e., when $k_{1}=0$ and $k_{2}=0$ ).

Hardware implementations of spiking neurons can be extremely useful for a large variety of applications including neuromorphic silicon neurons which consist of a hybrid analog/digital very large scale integration circuits that emulate the electrophysiological behavior of real neurons, conductances, and bi-dimensional generalized adaptive integrate and fire models that operate in real-time [110,111]. Neuromorphic silicon neurons offer a medium in which neuronal networks can be emulated directly in hardware rather than simply simulated on a general purpose computer. The mechanism of SISR investigated in this paper could find useful applications in the control of the effects of noise in neuromorphic silicon circuits.

Looking forward, we must be cognizant that Lévy white noise is only one possible type of a non-Gaussian white noise which can induce SISR. The mechanism via which noise with a temporal correlation (i.e., colored noise) can induce SISR is worth investigating. The additional timescale brought into the system by this temporal correlation may come along with new interesting dynamics.

Acknowledgements MEY acknowledges support from the Deutsche Forschungsgemeinschaft (DFG, German Research Foundation) - Project No. 456989199.

Funding Open Access funding enabled and organized by Projekt DEAL.

Data Availability Statement The simulation data that support the findings of this study are available within the article.

\section{Declarations}

Conflict of interest The authors declare that there is no conflict of interest in relation to this article.

Open Access This article is licensed under a Creative Commons Attribution 4.0 International License, which permits use, sharing, adaptation, distribution and reproduction in any medium or format, as long as you give appropriate credit to the original author(s) and the source, provide a link to the Creative Commons licence, and indicate if changes were made. The images or other third party material in this article are included in the article's Creative Commons licence, unless indicated otherwise in a credit line to the material. If material is not included in the article's Creative Commons licence and your intended use is not permitted by statutory regulation or exceeds the permitted use, you will need to obtain permission directly from the copyright holder. To view a copy of this licence, visit http://creativecommons.org/licenses/ by $/ 4.0 /$.

\section{Appendix}

The numerical solutions of Eq. (14) are obtained by the fourth-order stochastic Runge-Kutta algorithm given by: 


$$
\left\{\begin{array}{l}
A_{1}=f_{1}\left(v_{n}, w_{n}, \phi_{n}, t\right), \\
B_{1}=\varepsilon f_{2}\left(v_{n}, w_{n}, \phi_{n}, t\right), \\
C_{1}=\varepsilon f_{3}\left(v_{n}, w_{n}, \phi_{n}, t\right), \\
A_{2}=f_{1}\left(v_{n}+\frac{A_{1}}{2}, w_{n}+\frac{B_{1}}{2}, \phi_{n}+\frac{C_{1}}{2}, t+\frac{d t}{2}\right), \\
B_{2}=\varepsilon f_{2}\left(v_{n}+\frac{A_{1}}{2}, w_{n}+\frac{B_{1}}{2}, \phi_{n}+\frac{C_{1}}{2}, t+\frac{d t}{2}\right), \\
C_{2}=\varepsilon f_{3}\left(v_{n}+\frac{A_{1}}{2}, w_{n}+\frac{B_{1}}{2}, \phi_{n}+\frac{C_{1}}{2}, t+\frac{d t}{2}\right), \\
A_{3}=f_{1}\left(v_{n}+\frac{A_{2}}{2}, w_{n}+\frac{B_{2}}{2}, \phi_{n}+\frac{C_{2}}{2}, t+\frac{d t}{2}\right), \\
B_{3}=\varepsilon f_{2}\left(v_{n}+\frac{A_{2}}{2}, w_{n}+\frac{B_{2}}{2}, \phi_{n}+\frac{C_{2}}{2}, t+\frac{d t}{2}\right),(\mathrm{A} .1) \\
C_{3}=\varepsilon f_{3}\left(v_{n}+\frac{A_{2}}{2}, w_{n}+\frac{B_{2}}{2}, \phi_{n}+\frac{C_{2}}{2}, t+\frac{d t}{2}\right), \\
A_{4}=f_{1}\left(v_{n}+A_{3}, w_{n}+B_{3}, \phi_{n}+C_{3}, t+d t\right), \\
B_{4}=\varepsilon f_{2}\left(v_{n}+A_{3}, w_{n}+B_{3}, \phi_{n}+C_{3}, t+d t\right), \\
C_{4}=\varepsilon f_{3}\left(v_{n}+A_{3}, w_{n}+B_{3}, \phi_{n}+C_{3}, t+d t\right), \\
v_{n+1}=v_{n}+\frac{d t}{6}\left[A_{1}+2\left(A_{2}+A_{3}\right)+A_{4}\right]+d t(1 / \alpha) \hat{L}_{n}, \\
w_{n+1}=w_{n}+\frac{d t}{6}\left[B_{1}+2\left(B_{2}+B_{3}\right)+B_{4}\right], \\
\phi_{n+1}=\phi_{n}+\frac{d t}{6}\left[C_{1}+2\left(C_{2}+C_{3}\right)+C_{4}\right],
\end{array}\right.
$$

where $d t$ represents the time step and $\hat{L}_{n}$ is the Lévy distributed random variable (with the intensity $\sigma$ ) that we generate by using the Janicki-Weron algorithm [112] which has been proven $[113,114]$ to generate stable random variable for all admissible values of the parameters $\alpha, \beta \mu$, and $\sigma$.

\section{References}

1. Wiesenfeld, K., Moss, F.: Stochastic resonance and the benefits of noise: from ice ages to crayfish and squids. Nature 373, 33-36 (1995)

2. Douglass, J.K., Wilkens, L., Pantazelou, E., Moss, F.: Noise enhancement of information transfer in crayfish mechanoreceptors by stochastic resonance. Nature $\mathbf{3 6 5}$, 337-340 (1993)

3. Guo, D., Perc, M., Liu, T., Yao, D.: Functional importance of noise in neuronal information processing. EPL (Europhys. Lett.) 124, 50001 (2018)

4. Longtin, A.: Stochastic resonance in neuron models. J. Stat. Phys. 70, 309-327 (1993)

5. Gammaitoni, L., Hänggi, P., Jung, P., Marchesoni, F.: Stochastic resonance. Rev. Mod. Phys. 70, 223 (1998)

6. Wang, Z., Xu, Y., Yang, H.: Lévy noise induced stochastic resonance in an fhn model. Sci. China Technol. Sci. 59, 371-375 (2016)

7. Li, X., Ning, L.: Stochastic resonance in fizhugh-nagumo model driven by multiplicative signal and non-gaussian noise. Indian J. Phys. 89, 189-194 (2015)

8. Collins, J.J., Imhoff, T.T., Grigg, P.: Noise-enhanced information transmission in rat sal cutaneous mechanoreceptors via aperiodic stochastic resonance. J. Neurophysiol. 76, 642-645 (1996)

9. Nozaki, D., Mar, D.J., Grigg, P., Collins, J.J.: Effects of colored noise on stochastic resonance in sensory neurons. Phys. Rev. Lett. 82, 2402 (1999)
10. Guo, Y., Zhou, P., Yao, Z., Ma, J.: Biophysical mechanism of signal encoding in an auditory neuron. Nonlinear Dyn. 105, 3603-3614 (2021)

11. Lindner, B., Garcia-Ojalvo, J., Neiman, A., SchimanskyGeier, L.: Effects of noise in excitable systems. Phys. Rep. 392, 321-424 (2004)

12. Guo, D., Perc, M., Zhang, Y., Xu, P., Yao, D.: Frequencydifference-dependent stochastic resonance in neural systems. Phys. Rev. E 96, 022415 (2017)

13. Patel, A., Kosko, B.: Stochastic resonance in continuous and spiking neuron models with levy noise. IEEE Trans. Neural Netw. 19, 1993-2008 (2008)

14. Xu, Y., Guo, Y., Ren, G., Ma, J.: Dynamics and stochastic resonance in a thermosensitive neuron. Appl. Math. Comput. 385, 125427 (2020)

15. Pikovsky, A.S., Kurths, J.: Coherence resonance in a noisedriven excitable system. Phys. Rev. Lett. 78, 775 (1997)

16. Zhou, C., Kurths, J., Hu, B.: Array-enhanced coherence resonance: nontrivial effects of heterogeneity and spatial independence of noise. Phys. Rev. Lett. 87, 098101 (2001)

17. Neiman, A., Saparin, P.I., Stone, L.: Coherence resonance at noisy precursors of bifurcations in nonlinear dynamical systems. Phys. Rev. E 56, 270 (1997)

18. Zhu, J.: Phase sensitivity for coherence resonance oscillators. Nonlinear Dyn. 102, 2281-2293 (2020)

19. Carrillo, O., Santos, M.A., García-Ojalvo, J., Sancho, J.: Spatial coherence resonance near pattern-forming instabilities. EPL (Europhys. Lett.) 65, 452 (2004)

20. Perc, M.: Spatial coherence resonance in excitable media. Phys. Rev. E 72, 016207 (2005)

21. Gutkin, B., Jost, J., Tuckwell, H.: Transient termination of spiking by noise in coupled neurons. EPL (Europhys. Lett.) 81, 20005 (2007)

22. Gutkin, B.S., Jost, J., Tuckwell, H.C.: Inhibition of rhythmic neural spiking by noise: the occurrence of a minimum in activity with increasing noise. Naturwissenschaften 96, 1091-1097 (2009)

23. Yamakou, M.E., Jost, J.: Weak-noise-induced transitions with inhibition and modulation of neural oscillations. Biol. Cybern. 112, 445-463 (2018)

24. Uzuntarla, M., Cressman, J.R., Ozer, M., Barreto, E.: Dynamical structure underlying inverse stochastic resonance and its implications. Phys. Rev. E 88, 042712 (2013)

25. Yamakou, M.E., Jost, J.: A simple parameter can switch between different weak-noise-induced phenomena in a simple neuron model. EPL (Europhys. Lett.) 120, 18002 (2017)

26. Krauss, P., Prebeck, K., Schilling, A., Metzner, C.: Recurrence resonance in three-neuron motifs. Front. Comput. Neurosci. 13,(2019)

27. Muratov, C.B., Vanden-Eijnden, E., Weinan, E.: Selfinduced stochastic resonance in excitable systems. Phys. D Nonlinear Phenom. 210, 227-240 (2005)

28. DeVille, R.L., Vanden-Eijnden, E., Muratov, C.B.: Two distinct mechanisms of coherence in randomly perturbed dynamical systems. Phys. Rev. E 72, 031105 (2005)

29. Muratov, C.B., Vanden-Eijnden, E.: Noise-induced mixedmode oscillations in a relaxation oscillator near the onset of a limit cycle. Chaos An Interdis. J. Nonlinear Sci. 18, 015111 (2008) 
30. DeVille, R.L., Vanden-Eijnden, E.: A nontrivial scaling limit for multiscale markov chains. J. Stat. Phys. 126, 7594 (2007)

31. DeVille, R.L., Vanden-Eijnden, E., et al.: Self-induced stochastic resonance for brownian ratchets under load. Commun. Math. Sci. 5, 431-466 (2007)

32. Shen, J., Chen, L, Aihara, K.: Self-induced stochastic resonance in microrna regulation of a cancer network. In: The fourth international conference on computational systems biology, pp. 251-257 (2010)

33. Yamakou, M.E., Jost, J.: Coherent neural oscillations induced by weak synaptic noise. Nonlinear Dyn. 93, 21212144 (2018)

34. Yamakou, M.E., Jost, J.: Control of coherence resonance by self-induced stochastic resonance in a multiplex neural network. Phys. Rev. E 100, 022313 (2019)

35. Yamakou, M.E., Hjorth, P.G., Martens, E.A.: Optimal selfinduced stochastic resonance in multiplex neural networks: electrical vs. chemical synapses. Front. Comput. Neurosci. 14, 62 (2020)

36. Wu, J., Xu, Y., Ma, J.: Lévy noise improves the electrical activity in a neuron under electromagnetic radiation. PLoS One 12, e0174330 (2017)

37. Nurzaman, S.G., Matsumoto, Y., Nakamura, Y., Shirai, K., Koizumi, S., Ishiguro, H.: From lévy to brownian: a computational model based on biological fluctuation. PloS one 6, e16168 (2011)

38. Peng, C.-K., Mietus, J., Hausdorff, J., Havlin, S., Stanley, H.E., Goldberger, A.L.: Long-range anticorrelations and non-gaussian behavior of the heartbeat. Phys. Rev. Lett. 70, 1343 (1993)

39. Mantegna, R.N., Stanley, H.E.: Scaling behaviour in the dynamics of an economic index. Nature 376, 46-49 (1995)

40. Shlesinger, M.F., Zaslavsky, G.M., Klafter, J.: Strange kinetics. Nature 363, 31-37 (1993)

41. Segev, R., Benveniste, M., Hulata, E., Cohen, N., Palevski, A., Kapon, E., Shapira, Y., Ben-Jacob, E.: Long term behavior of lithographically prepared in vitro neuronal networks. Phys. Rev. Lett. 88, 118102 (2002)

42. Rocha, E.G., Santos, E.P., dos Santos, B.J., Samuel, S., Pincheira, P.I., Argolo, C., Moura, A.L.: Lévy flights for light in ordered lasers. Phys. Rev. A 101, 023820 (2020)

43. Novikov, D.S., Drndic, M., Levitov, L., Kastner, M., Jarosz, M., Bawendi, M.: Lévy statistics and anomalous transport in quantum-dot arrays. Phys. Rev. B 72, 075309 (2005)

44. Lisowski, B., Valenti, D., Spagnolo, B., Bier, M., Gudowska-Nowak, E.: Stepping molecular motor amid lévy white noise. Phys. Rev. E 91, 042713 (2015)

45. Stanley, H.E., Mantegna, R.N.: An introduction to econophysics. Cambridge University Press, Cambridge (2000)

46. Barndorff-Nielsen, O.E., Shephard, N.: Non-gaussian ornstein-uhlenbeck-based models and some of their uses in financial economics. J. Royal Stat. Soc. Ser. B (Stat. Methodol.) 63, 167-241 (2001)

47. Perc, M.: Transition from gaussian to levy distributions of stochastic payoff variations in the spatial prisoner's dilemma game. Phys. Rev. E 75, 022101 (2007)

48. Dubkov, A.A., Spagnolo, B., Uchaikin, V.V.: Lévy flight superdiffusion: an introduction. Int. J. Bifurc. Chaos 18, 2649-2672 (2008)
49. Xu, Y., Li, Y., Zhang, H., Li, X., Kurths, J.: The switch in a genetic toggle system with lévy noise. Sci. Rep. 6, 1-11 (2016)

50. Feng, J., Xu, W., Xu, Y., Wang, X.: Effects of lévy noise in a neuronal competition model. Phys. A Stat. Mech. Appl. 531, 121747 (2019)

51. Guo, Y., Wang, L., Dong, Q., Lou, X.: Dynamical complexity of fitzhugh-nagumo neuron model driven by lévy noise and gaussian white noise. Math. Comput. Simul. 181, 430-443 (2021)

52. Semenova, N., Zakharova, A.: Weak multiplexing induces coherence resonance. Chaos An Interdis. J. Nonlinear Sci. 28, 051104 (2018)

53. Lv, M., Wang, C., Ren, G., Ma, J., Song, X.: Model of electrical activity in a neuron under magnetic flow effect. Nonlinear Dyn. 85, 1479-1490 (2016)

54. Chua, L.: Memristor-the missing circuit element. IEEE Trans. Circuit Theory 18, 507-519 (1971)

55. Yamakou, M.E.: Chaotic synchronization of memristive neurons: Lyapunov function versus Hamilton function. Nonlinear Dyn. 101, 487-500 (2020)

56. Wu, F., Wang, C., Jin, W., Ma, J.: Dynamical responses in a new neuron model subjected to electromagnetic induction and phase noise. Phys. A Stat. Mech. Appl. 469, 81-88 (2017)

57. Ma, J., Mi, L., Zhou, P., Xu, Y., Hayat, T.: Phase synchronization between two neurons induced by coupling of electromagnetic field. Appl. Math. Comput. 307, 321-328 (2017)

58. Xu, Y., Jia, Y., Ma, J., Hayat, T., Alsaedi, A.: Collective responses in electrical activities of neurons under field coupling. Sci. Rep. 8, 1-10 (2018)

59. Ma, J., Wu, F., Hayat, T., Zhou, P., Tang, J.: Electromagnetic induction and radiation-induced abnormality of wave propagation in excitable media. Phys. A Stat. Mech. Appl. 486, 508-516 (2017)

60. Wu, F., Wang, C., Xu, Y., Ma, J.: Model of electrical activity in cardiac tissue under electromagnetic induction. Sci. Rep. 6, 1-12 (2016)

61. Yao, Z., Ma, J., Yao, Y., Wang, C.: Synchronization realization between two nonlinear circuits via an induction coil coupling. Nonlinear Dyn. 96, 205-217 (2019)

62. Zhang, Y., ChunNi, W., Jun, T., Jun, M., GuoDong, R.: Phase coupling synchronization of fhn neurons connected by a josephson junction. Sci. China Technol. Sci. 63, 23282338 (2020)

63. Wang, C., Lv, M., Alsaedi, A., Ma, J.: Synchronization stability and pattern selection in a memristive neuronal network. Chaos An Interdis. J. Nonlinear Sci. 27, 113108 (2017)

64. Ma, J., Wu, F., Wang, C.: Synchronization behaviors of coupled neurons under electromagnetic radiation. Int. J. Mod. Phys. B 31, 1650251 (2017)

65. Njitacke, Z.T., Doubla, I.S., Mabekou, S., Kengne, J.: Hidden electrical activity of two neurons connected with an asymmetric electric coupling subject to electromagnetic induction: coexistence of patterns and its analog implementation. Chaos Solit. Fract. 137, 109785 (2020)

66. Wouapi, M.K., Fotsin, B.H., Ngouonkadi, E.B.M., Kemwoue, F.F., Njitacke, Z.T.: Complex bifurcation analysis and synchronization optimal control for hindmarsh-rose 
neuron model under magnetic flow effect. Cognit. Neurodyn. 15, 315-347 (2021)

67. Wouapi, K.M., Fotsin, B.H., Louodop, F.P., Feudjio, K.F., Njitacke, Z.T., Djeudjo, T.H.: Various firing activities and finite-time synchronization of an improved hindmarsh-rose neuron model under electric field effect. Cognit. Neurodyn. 14, 375-397 (2020)

68. Jin, W., Wang, A., Ma, J., Lin, Q.: Effects of electromagnetic induction and noise on the regulation of sleep wake cycle. Sci. China Technol. Sci. 62, 2113-2119 (2019)

69. FitzHugh, R.: Mathematical models of excitation and propagation in nerve. Biological engineering , 1-85 (1969)

70. Xu, B., Binczak, S., Jacquir, S., Pont, O., Yahia, H.: Parameters analysis of fitzhugh-nagumo model for a reliable simulation, In: 36th annual international conference of the ieee engineering in medicine and biology society. IEEE 2014, 4334-4337 (2014)

71. Kuehn, C.: Multiple Time Scale Dynamics. Springer, Berlin (2015)

72. Wu, F., Ma, J., Zhang, G.: A new neuron model under electromagnetic field. Appl. Math. Comput. 347, 590-599 (2019)

73. Bao, B., Liu, Z., Xu, J.: Steady periodic memristor oscillator with transient chaotic behaviours. Electron. Lett. 46, 237-238 (2010)

74. Muthuswamy, B.: Implementing memristor based chaotic circuits. Int. J. Bifurc. Chaos 20, 1335-1350 (2010)

75. Li, Q., Zeng, H., Li, J.: Hyperchaos in a 4d memristive circuit with infinitely many stable equilibria. Nonlinear Dyn. 79, 2295-2308 (2015)

76. Ma, J., Wang, Y., Wang, C., Xu, Y., Ren, G.: Mode selection in electrical activities of myocardial cell exposed to electromagnetic radiation. Chaos Solit. Fract. 99, 219-225 (2017)

77. Hong, Q.-H., Zeng, Y.-C., Li, Z.-J.: Design and simulation of chaotic circuit for flux-controlled memristor and chargecontrolled memristor (2013)

78. Sato, K.-I., Ken-Iti, S., Katok, A.: Lévy processes and infinitely divisible distributions. Cambridge University Press, Cambridge (1999)

79. Bertoin, J.: Lévy processes. Cambridge University Press, Melbourne, NY (1996)

80. Dybiec, B., Gudowska-Nowak, E.: Lévy stable noiseinduced transitions: stochastic resonance, resonant activation and dynamic hysteresis. J. Stat. Mech. Theory Exp. 2009, P05004 (2009)

81. Dybiec, B., Gudowska-Nowak, E., Hänggi, P.: Escape driven by $\alpha$-stable white noises. Phys. Rev. E 75, 021109 (2007)

82. Prokhorov, Y.V., Feller, W.: An introduction to probability theory and its applications. Teoriya Veroyatnostei i ee Primeneniya 10, 204-206 (1965)

83. Penson, K., Górska, K.: Exact and explicit probability densities for one-sided lévy stable distributions. Phys. Rev. Lett. 105, 210604 (2010)

84. Górska, K., Penson, K.: Lévy stable two-sided distributions: exact and explicit densities for asymmetric case. Phys. Rev. E 83, 061125 (2011)

85. Izhikevich, E.M.: Neural excitability, spiking and bursting. Int. J. Bifurc. Chaos 10, 1171-1266 (2000)
86. Ding, D., Jiang, L., Hu, Y., Yang, Z., Li, Q., Zhang, Z., Wu, Q.: Hidden coexisting firings in fractional-order hyperchaotic memristor-coupled hr neural network with two heterogeneous neurons and its applications. Chaos An Interdis. J. Nonlinear Sci. 31, 083107 (2021)

87. Yan, B., Panahi, S., He, S., Jafari, S.: Further dynamical analysis of modified fitzhugh-nagumo model under the electric field. Nonlinear Dyn. 101, 521-529 (2020)

88. Rocsoreanu, C., Georgescu, A., Giurgiteanu, N.: The FitzHugh-Nagumo model: bifurcation and dynamics, vol. 10. Springer, New York (2012)

89. Yamakou, M.E.: Weak-noise-induced phenomena in a slow-fast dynamical system, Ph.D. thesis, Max Planck Institute for Mathematics in the Sciences, Max Planck Society, (2018)

90. Kuehn, C.: Multiple time scale dynamics, vol. 191. Springer, New York (2015)

91. Chechkin, A.V., Sliusarenko, O.Y., Metzler, R., Klafter, J.: Barrier crossing driven by Lévy noise: Universality and the role of noise intensity. Phys. Rev. E 75, 041101 (2007)

92. Imkeller, P., Pavlyukevich, I.: Lévy flights: transitions and meta-stability. J. Phys. A Math. Gen. 39, L237-L246 (2006)

93. Kramers, H.A.: Brownian motion in a field of force and the diffusion model of chemical reactions. Physica 7, 284-304 (1940)

94. Stambaugh, C., Chan, H.B.: Noise-activated switching in a driven nonlinear micromechanical oscillator. Phys. Rev. B 73, 172302 (2006)

95. Koren, T., Lomholt, M.A., Chechkin, A.V., Klafter, J., Metzler, R.: Leapover lengths and first passage time statistics for lévy flights. Phys. Rev. Lett. 99, 160602 (2007)

96. Dybiec, B., Gudowska-Nowak, E., Chechkin, A.: To hit or to pass it over-remarkable transient behavior of first arrivals and passages for lévy flights in finite domains. J. Phys. A Math. Theor. 49, 504001 (2016)

97. Ditlevsen, P.D.: Anomalous jumping in a double-well potential. Phys. Rev. E 60, 172 (1999)

98. Pei, X., Wilkens, L., Moss, F.: Noise-mediated spike timing precision from aperiodic stimuli in an array of hodgekinhuxley-type neurons. Phys. Rev. Lett. 77, 4679 (1996)

99. Gabbiani, F., Koch, C.: Principles of spike train analysis. Methods Neuronal Model. 12, 313-360 (1998)

100. Perina, J.: Coherence of light. Springer, New York (1985)

101. Saleh, B.: Photoelectron Statistics. Springer Series in Optical Sciences, vol. 6. Springer, Berlin Heidelberg, Berlin, Heidelberg (1978)

102. Huang, J., Tao, W., Xu, B.: Effects of small time delay on a bistable system subject to lévy stable noise. J. Phys. A Math. Theor. 44, 385101 (2011)

103. Xu, Y., Li, J., Feng, J., Zhang, H., Xu, W., Duan, J.: Lévy noise-induced stochastic resonance in a bistable system. The Eur. Phys. J. B 86, 1-7 (2013)

104. Rümelin, W.: Numerical treatment of stochastic differential equations. SIAM J. Num. Anal. 19, 604-613 (1982)

105. Wilkie, J.: Numerical methods for stochastic differential equations. Phys. Rev. E 70, 017701 (2004)

106. Burrage, K., Burrage, P., Higham, D.J., Kloeden, P.E., Platen, E.: Comment on Numerical methods for stochastic differential equations. Phys. Rev. E 74, 068701 (2006) 
107. Kosko, B., Mitaim, S.: Robust stochastic resonance: signal detection and adaptation in impulsive noise. Phys. Rev. E 64, 051110 (2001)

108. Mitaim, S., Kosko, B.: Adaptive stochastic resonance in noisy neurons based on mutual information. IEEE Trans. Neural Netw. 15, 1526-1540 (2004)

109. Liu, R.-N., Kang, Y.-M.: Stochastic resonance in underdamped periodic potential systems with alpha stable lévy noise. Phys. Lett. A 382, 1656-1664 (2018)

110. Indiveri, G., Linares-Barranco, B., Hamilton, T.J., Van Schaik, A., Etienne-Cummings, R., Delbruck, T., Liu, S.C., Dudek, P., Häfliger, P., Renaud, S., et al.: Neuromorphic silicon neuron circuits. Front. Neurosci. 5, 73 (2011)

111. Covi, E., George, R., Frascaroli, J., Brivio, S., Mayr, C., Mostafa, H., Indiveri, G., Spiga, S.: Spike-driven thresholdbased learning with memristive synapses and neuromorphic silicon neurons. J. Phys. D Appl. Phys. 51, 344003 (2018)
112. Janicki, A., Weron, A.: Simulation and chaotic behavior of alpha-stable stochastic processes, vol. 178. CRC Press, Boca Raton (1993)

113. Zolotarev, V.: One dimensional stable distributions (american mathematical society, providence, ri), Russian original (1983)

114. Weron, R.: On the chambers-mallows-stuck method for simulating skewed stable random variables. Stat. Probab. Lett. 28, 165-171 (1996)

Publisher's Note Springer Nature remains neutral with regard to jurisdictional claims in published maps and institutional affiliations. 